\title{
Molecular characteristics of S-RNase alleles as the determinant of self-incompatibility in the style of Fragaria viridis
}

\author{
Jianke Du ${ }^{1,2}$, Chunfeng Ge ${ }^{1,3}$, Tingting $\mathrm{Li}^{1}$, Sanhong Wang ${ }^{1}$, Zhihong Gao ${ }^{1}$, Hidenori Sassa ${ }^{2}$ and Yushan Qiao $\mathbb{D}^{1 凶}$
}

\begin{abstract}
Strawberry (Fragaria spp.) is a member of the Rosoideae subfamily in the family Rosaceae. The self-incompatibility (SI) of some diploid species is a key agronomic trait that acts as a basic pollination barrier; however, the genetic mechanism underlying $\mathrm{SI}$ control in strawberry remains unclear. Two candidate $S$-RNases $\left(S_{a}\right.$ - and $S_{b}$-RNase) identified in the transcriptome of the styles of the self-incompatible Fragaria viridis 42 were confirmed to be $\mathrm{SI}$ determinants at the $\mathrm{S}$ locus following genotype identification and intraspecific hybridization using selfing progenies. Whole-genome collinearity and RNase T2 family analysis revealed that only an S locus exists in Fragaria; however, none of the compatible species contained S-RNase. Although the results of interspecific hybridization experiments showed that $F$. viridis (SI) styles could accept pollen from F. mandshurica (self-compatible), the reciprocal cross was incompatible. $S_{a}$ and $S_{b}$-RNase contain large introns, and their noncoding sequences (promotors and introns) can be transcribed into long noncoding RNAs (IncRNAs). Overall, the genus Fragaria exhibits S-RNase-based gametophytic SI, and S-RNase loss occurs at the $S$ locus of compatible germplasms. In addition, a type of SI-independent unilateral incompatibility exists between compatible and incompatible Fragaria species. Furthermore, the large introns and neighboring IncRNAs in $S$-RNase in Fragaria could offer clues about S-RNase expression strategies.
\end{abstract}

\section{Introduction}

The germplasm of low ploidy wild strawberry contains abundant genetic resources that control valuable traits and is a potential resource for improving cultivated strawberry. However, pollination obstacles between styles and pollen have considerably hampered strawberry breeding $^{1-6}$. Incompatibility includes self-incompatibility (SI) and interspecific incompatibility, and it has been demonstrated that both have common genetic factors and share some intermediate mechanisms in Solanaceae ${ }^{7,8}$. SI is a type of pollination barrier that serves as a basis for studying other incompatibility mechanisms ${ }^{7,8}$. Therefore, the elucidation of the molecular mechanism underlying SI

Correspondence: Yushan Qiao (qiaoyushan@njau.edu.cn)

'Laboratory of Fruit Crop Biotechnology, College of Horticulture, Nanjing Agricultural University, Nanjing 210095 Jiangsu, China

${ }^{2}$ Laboratory of Genetics and Plant Breeding, Graduate School of Horticulture, Chiba University, Matsudo 271-8510 Chiba, Japan

Full list of author information is available at the end of the article will enable the development of strategies aimed at improving molecular breeding in strawberry.

Gametophytic SI (GSI) is a genetic mechanism that inhibits the self-pollination and growth of inbred pollen tubes. The phenotype is determined by the $\mathrm{S}$ haplotype of the pollen, which results in the generation of samegenotype pollen tubes that cannot normally extend to the ovary in the style; this process may involve multiple genetic mechanisms. To date, two GSI mechanisms have been identified, including $S$-RNase-based GSI, identified in Solanaceae, Plantaginaceae, and Rosaceae, and signal transduction GSI, identified in Papaveraceae ${ }^{7,10,11}$. Strawberries belong to the genus Fragaria in the subfamily Rosoideae (family Rosaceae). Although there is broad consensus that the family Rosaceae is divided into three subfamilies (Dryadoideae, Rosoideae, and Amygdaloideae), the genetic relationships among the subfamilies remain controversial ${ }^{12-14}$. The tribes Maleae (which includes Malus and Pyrus) and Amygdaleae (which

\section{(c) The Author(s) 2021}

(c) (i) Open Access This article is licensed under a Creative Commons Attribution 4.0 International License, which permits use, sharing, adaptation, distribution and reproduction cc) in any medium or format, as long as you give appropriate credit to the original author(s) and the source, provide a link to the Creative Commons license, and indicate if changes were made. The images or other third party material in this article are included in the article's Creative Commons license, unless indicated otherwise in a credit line to the material. If material is not included in the article's Creative Commons license and your intended use is not permitted by statutory regulation or exceeds the permitted use, you will need to obtain permission directly from the copyright holder. To view a copy of this license, visit http://creativecommons.org/licenses/by/4.0/. 
includes Prunus) in the subfamily Amygdaloideae exhibit $S$-RNase-based GSI, and its mechanism has been studied extensively. Among them, the genera Malus and Pyrus have similar GSI mechanisms that are distinct from the mechanism in the genus Prunus ${ }^{15}$. However, little is known about the SI mechanism in the genus Fragaria in the subfamily Rosoideae ${ }^{6,16-19}$.

Although Rosaceae members exhibit the $S$-RNase-based GSI system, some self-compatible variants exist in nature $^{16,17}$. Recently, increased research on SI has helped unravel the mechanisms by which some mutations exert their effects. The major reasons for the occurrence of selfcompatibility (SC) include the sequence loss of key genes ${ }^{20,21}$ or the loss of function of S-encoded proteins because of sequence variations at the $S$ locus $^{22,23}$. SI requires a certain $S$-RNase threshold, and the abnormal expression of $S$-RNase can overcome the SI in a spe$\mathrm{cies}^{24,25}$. There are some indications that the $S$ intron sequence and $S$ locus methylation level can influence the expression of $S$-RNase ${ }^{26,27}$. Flowering plants of the same species, including strawberry, can exhibit both SI and SC. Unilateral incompatibility (UI) often exists between the two types and is considered to be related to $\mathrm{SI}^{28}$. In addition, the UI observed in $\mathrm{SC} \times \mathrm{SC}$ crosses supports the existence of additional interspecific barriers that are not dependent on $\mathrm{SI}^{7,8}$. The reasons underlying the compatibility of strawberries have not been elucidated, and to our knowledge, there have been no studies on whether there is a types of UI between SI and SC species in Fragaria that is not associated with $S$-RNase-based SI.

Fragaria spp. exhibit SI, with Fragaria viridis, F. nubicola, F. pentaphylla, and F. nipponica exhibiting SI and $F$. vesca and $F$. nilgerrensis exhibiting $\mathrm{SC}^{1,6,29-31}$. Bosković et al. ${ }^{6}$ suggested that there are two unlinked RNase loci that control strawberry incompatibility that are different from the single $S$ locus in Amygdaloideae ${ }^{17}$. The presence of any locus can result in the specific rejection of cognate pollen, in addition to the nonspecific rejection of pollen with $S n$ and $T n$ null allele genotypes. The polypeptides encoded by the two loci are also different from those in the Amygdaloideae subfamily. The above results are based on analyses of style RNase; however, not all RNases expressed in styles are $S$-RNase ${ }^{6,18}$. Therefore, whether there are two $\mathrm{S}$ control loci still requires further verification. Similarly, $S$-RNases that determine the style type in incompatible species of the genus Fragaria need to be explored further.

It is speculated that the genus Fragaria exhibits $S$ RNase-based SI similar to that in the Maleae and Amygdaleae tribes ${ }^{6,18}$. In the present study, two S-RNase candidates $\left(S_{a}, S_{b}\right.$-RNase) were obtained from the style expression database of $F$. viridis 42 . The sequence and expression analysis of the two candidates, the identification of the genotypes of selfing lines, and intraspecific hybridization between different $\mathrm{S}$ genotype lines confirmed that $S_{a}$ and $S_{b}$-RNase are alleles and style SI determinants in $F$. viridis. Expression analysis of S-RNase and the whole-genome identification of the RNase T2 family genes showed that S-RNase was lost in selfcompatible strawberry germplasms. Only one $S$ locus was found on chromosome 6 based on collinearity analysis using the $F$. vesca genome and RNase T2 family genes analysis in $F$. viridis. Interspecific hybridization revealed a principle inconsistent with the SI $\times$ SC rule in Fragaria plants, in addition to having no correlation with the $S$ RNase genotype. Furthermore, UI was observed between compatible strawberries. In addition, the $S$-RNase of the genus Fragaria is a very large gene $\left(S_{a}\right.$ and $S_{b}$-RNase are 30 and $23 \mathrm{~kb}$, respectively), and the noncoding sequence of the genes (intron and promotor) can transcribe long noncoding RNAs (lncRNAs); these may provide new insights into the mechanism of the regulation of $S$-RNase expression.

\section{Results}

Screening and analysis of $S$-RNase candidate genes

Screening of RNase T2 family genes in F. viridis and $F$. vesca

$S$-RNase belongs to Class III in the RNase T2 protein family $^{32}$. In contrast to non-S-RNase genes, $S$-RNase, as a candidate SI determinant, has specific characteristics that distinguish it from other RNase genes ${ }^{11,15,18}$. RNase T2 family gene members were screened using HMM, and 14 and 13 genes were screened from the proteome database in $F$. vesca and $F$. viridis, respectively. Detailed information about the characteristics of the selected RNase T2 family genes is shown in Table 1 . Based on this information, SI determinant candidates in $F$. viridis were selected, and SC analysis in $F$. vesca was performed. Flower balls containing styles from wild $F$. viridis 42 at $0 \mathrm{~h}$ and $24 \mathrm{~h}$ after self-pollination were selected for transcriptome sequencing and for further use in the establishment of a style proteome database. The basic data used to obtain the proteome data for $F$. viridis have been submitted to the Sequence Read Archive database. The accession numbers are PRJNA361176, PRJNA361185, PRJNA361192, PRJNA361199, PRJNA361204, and PRJNA361208.

\section{$S$-RNase prediction in $F$. viridis and $F$. vesca}

The presence of a signal peptide is one of the prerequisites for the extracellular secretion of proteins $^{22,33,34}$, and 12 and 8 deduced proteins containing signal peptides were obtained from $F$. vesca and $F$. viridis, respectively. The functional domain of RNase T2 is a basic structure that contains essential functional units and plays a key role in mediating GSI responses ${ }^{35-38}$. We set a domain integrity $>60 \%$ as the condition for screening $S$-RNase and further filtered 3 and 1 
Table 1 Characteristics of the RNase T2 gene family in $F$. vesca and $F$. viridis

\begin{tabular}{|c|c|c|c|c|c|c|c|c|}
\hline Gene ID & $\begin{array}{l}\text { Chromosome } \\
\text { location }\end{array}$ & Intron number & pl & Molecular weight & $\begin{array}{l}\text { Signal } \\
\text { peptide }\end{array}$ & Domain size & $\begin{array}{l}\text { Number of } \\
\text { amino acids }\end{array}$ & Pattern 4 \\
\hline FvH4_4g31300.1 & Chr4:30502482 & 7 & 5.38 & 102.0 & $\mathrm{~N}$ & $>60 \%$ & 916 & CPSSNG \\
\hline FvH4_4g31290.1 & Chr4:30499449 & 3 & 5.23 & 25.7 & Y23 & $>60 \%$ & 229 & CPSSNG \\
\hline FvH4_2g17310.1 & Chr2:14946893 & 7 & 8.17 & 27.6 & Y28 & $>60 \%$ & 245 & - \\
\hline FvH4_5g24800.1 & Chr5:16134164 & 3 & 9.37 & 26.6 & Y26 & $>60 \%$ & 239 & - \\
\hline FvH4_1g10040.1 & Chr1:5448902 & 1 & 8.08 & 26.0 & Y19 & $>60 \%$ & 230 & - \\
\hline FvH4_6g07740.1 & Chr6:4649328 & 1 & 6.21 & 25.2 & Y21 & $>60 \%$ & 220 & - \\
\hline FvH4_6g07690.1 & Chr6:4634036 & 1 & 6.28 & 26.2 & Y15 & $>60 \%$ & 226 & - \\
\hline FvH4_5g33850.1 & Chr5:24565949 & 1 & 8.07 & 31.3 & Y43 & $>60 \%$ & 270 & - \\
\hline FvH4_1g19170.1 & Chr1:11366424 & 1 & 8.95 & 22.7 & Y23 & $>60 \%$ & 198 & - \\
\hline FvH4_4g18130.1 & Chr4:22047584 & 1 & 8.09 & 27.7 & Y19 & $>60 \%$ & 241 & - \\
\hline FvH4_2g25650.1 & Chr2: 20739797 & 3 & 9.39 & 25.6 & N & $<60 \%$ & 223 & - \\
\hline FvH4_6g22290.1 & Chr6: 15948673 & 1 & 7.66 & 27.4 & Y17 & $<60 \%$ & 238 & - \\
\hline FvH4_2g25620.1 & Chr2: 20733334 & 2 & 9.81 & 15.5 & Y20 & $<60 \%$ & 133 & - \\
\hline FvH4_5g24550.1 & Chr5: 15908338 & 2 & 8.55 & 12.3 & Y26 & $<60 \%$ & 109 & - \\
\hline Unigene 18150.1 & Chr4:30504261 & 2 & 4.58 & 25.2 & Y27 & $>60 \%$ & 231 & CPSSSG \\
\hline Unigene13465.1 & Chr4:30500729 & 3 & 5.35 & 25.8 & Y23 & $>60 \%$ & 229 & CPSSNG \\
\hline Unigene11523.1 & Chr2:14950589 & 6 & 5.76 & 30.5 & Y29 & $>60 \%$ & 278 & - \\
\hline Unigene23139.1 & Chr6:5067585 & 1 & 8.53 & 26.2 & Y26 & $>60 \%$ & 228 & - \\
\hline CL6424.Contig1.1 & Chr1:5449834 & 1 & 8.57 & 25.8 & Y19 & $>60 \%$ & 229 & - \\
\hline Unigene10929.1 & $\mathrm{nr}$. & 2 & 8.68 & 25.2 & Y23 & $>60 \%$ & 218 & - \\
\hline Unigene7320.1 & nr. & 2 & 8.55 & 25.6 & Y24 & $>60 \%$ & 221 & - \\
\hline Unigene9248.2 & Chr5:24566583 & 0 & 5.47 & 12.4 & $\mathrm{~N}$ & $<60 \%$ & 104 & - \\
\hline Unigene638.1 & Chr6:4649354 & 1 & 5.26 & 12.4 & Y19 & $<60 \%$ & 109 & - \\
\hline Unigene31475.1 & Chr6:4634931 & 1 & 6.88 & 8.8 & N & $<60 \%$ & 79 & - \\
\hline Unigene23536.2 & Chr1:4771772 & 0 & 6.52 & 5.6 & $\mathrm{~N}$ & $<60 \%$ & 50 & - \\
\hline Unigene27236.1 & Chr2:14949271 & 0 & 5.86 & 9.1 & N & $<60 \%$ & 79 & - \\
\hline CL6424.Contig2.2 & Chr1:5449478 & 1 & 8.94 & 8.5 & $N$ & $<60 \%$ & 75 & - \\
\hline
\end{tabular}

The genes with a "FvH4" prefix are from $F$. vesca, and those with " $\mathrm{CL}$ " or "Unigene" prefixes are from $F$. viridis. The ".1" suffix represents the first transcript of the gene in $F$. vesca, and the ".1" and ".2" suffixes represent the first and second open reading frames (ORFs) of the gene, respectively, in $F$. viridis. All identified genes of the RNase T2 family from $F$. vesca were consistent with the results of a previous screening ${ }^{43}$. The gene position and intron number for $F$. vesca were obtained according to the GFF file (F. vesca genome_v4.0.a1). The position information for $F$. viridis was determined mainly based on the $F$. vesca genome_v4.0.a1 reference and was located using BLASTn. Intron number and position analyses were based on the characteristic structure of the intron boundary (the intron analysis of only Unigene23139.1 refers to the F. nilgerrensis genome, v1.0). "nr." means that the location information could not be obtained. In the signal peptide column, "N" indicates a lack of signal peptide information, " $Y$ " indicates the presence of signal peptide information, and the number indicates the size of the signal peptide. The domain size is represented as a percentage, which is the percentage of the RNase T2 domain contained in the selected protein within the full-length domain. Pattern 4 (RNase T2 lineage amino acid pattern 4) is a type of amino acid sequence; if an RNase T2 family member contains the pattern 4, it is not an S-RNase or S-lineage gene ${ }^{18}$. The domain analysis of the RNase T2 family genes in F. vesca and F. viridis (see Supplementary Fig. S1) and chromosomal localization information are presented in Supplementary Figs. S2 and S3, respectively. The size of the gene and the gene structure are shown in Supplementary Fig. S4. The intron position and number for Unigene10929.1 and Unigene7320.1 were analyzed, as shown in Supplementary Figs. S5, S6, and Supplementary Dataset S1.

nonstandard proteins in $F$. vesca and $F$. viridis, respectively. The isoelectric point (pI) of $S$-RNase has been reported to range from 8 to $10^{18,32,39,40}$. Based on the pI, three genes were simultaneously eliminated from $F$. vesca and $F$. viridis based on the last filtered result. As the number of S-RNase introns does not exceed two ${ }^{39,41}$, four proteins were obtained from $F$. vesca (FvH4_1g10040.1, FvH4_5g33850.1, FvH4_1g19170.1, and FvH4_4g18130.1), and four were obtained from $F$. viridis (Unigene 23139.1, CL6424.Contig1.1, Unigene 10929.1, and Unigene7320.1). S-RNase exhibits a high degree of polymorphism, with amino acid identity 
ranging from 30 to $90 \%^{11,16,37,42}$. Further identity analysis of the four selected proteins in $F$. viridis revealed two combinations that satisfied the conditions. The amino acid similarity between Unigene10929.1 and Unigene7320.1 was $56.48 \%$, and that between Unigene23139.1 and Unigene7320.1 was 30.88\% (Supplementary Table S1). The similarity between $S$ RNases within the same genus is known to be higher than that between $S$-RNases of different tribes ${ }^{35,37}$; this applied only for Unigene10929.1 and Unigene7320.1 in this study (Supplementary Table S1). The amino acid similarity of the four obtained $F$. vesca proteins with Unigene10929.1 and Unigene7320.1 was very low $(<30 \%)$. Unigene10929.1 and Unigene7320.1 were confirmed as the RNase T2s linked to the SI phenotype (see the section "Intraspecific hybridization between different $S$ genotype lines of $F$. viridis"); by extension, there were no eligible $S$-RNases in $F$. vesca. In addition, unlike the RNase T2 family genes that were assessed to be non- $S$ RNases, the position on the chromosome, the number of amino acids, and the amino acid sequence pattern indicated that Unigene10929.1 and Unigene7320.1 are candidate $S$-RNases ${ }^{6,16,39,41}$.

\section{Evolutionary analysis of RNase T2 family members}

A total of 105 known S-RNases (Supplementary Table S2), including 6, 37, 21, and 41 from Solanaceae, Malus, Pyrus, and Prunus, respectively, and 23 RNase T2 family members from $F$. vesca and $F$. viridis (Table 1), which can encode long amino acid sequences ( $>100$ amino acids), were selected for evolutionary analyses (Fig. 1). In contrast to $S$-RNase-like genes and $S$-RNase lineage genes ${ }^{18}, S$ RNase genes are evolutionarily orthologous ${ }^{18,35,37}$. Therefore, the evolutionary analysis indicated that the $S$ RNase candidates Unigene10929.1 and Unigene7320.1 experienced evolutionary pathways relatively similar to those of the Prunus S-RNase.

\section{Conservative sequence analysis of $S_{a}$ and $S_{b}$-RNase in F. viridis}

According to the transcript information, full-length specific primers were designed for the genes; CDS, $\mathrm{FS}_{\mathrm{a}} \mathrm{CDS}$, and $\mathrm{RS}_{\mathrm{a}} \mathrm{CDS}$ were used to obtain Unigene10929.1, and $\mathrm{FS}_{\mathrm{b}} \mathrm{CDS}$ and $\mathrm{RS}_{\mathrm{b}} \mathrm{CDS}$ were used to obtain Unigene7320.1 (Supplementary Table S3). The PCR products were obtained using style cDNA as the template and were further sequenced. The sequences of both were identical to those obtained from the transcriptome. The nucleotide similarity of both candidates was $71.43 \%$ (Supplementary Fig. S5). Using Prunus as a reference, the results of the comparison of the deduced amino acids showed that Unigene10929.1 and Unigene7320.1 had five characteristic conserved $S$-RNase regions $(\mathrm{C} 1, \mathrm{C} 2, \mathrm{C} 3, \mathrm{RC} 4$, and $\mathrm{C} 5)$ and a hypervariable region (RHV) (Fig. 1) and had similar amino acid differences in the regions between $\mathrm{C} 1$ and $\mathrm{C} 2$ and between $\mathrm{RC} 4$ and $\mathrm{C} 5$, in addition to similar intron locations and numbers $^{16,44}$ (Fig. 1; Supplementary Fig. S6).

In summary, Unigene10929.1 and Unigene7320.1 conformed to the $S$-RNase and allelic similarity characteristics and were named $S_{a}$-RNase and $S_{b}$-RNase, respectively, for experimental verification. The $S_{a}$-RNase and $S_{b}$-RNase sequences were deposited in GenBank (accession numbers MW223017 and MW223018, respectively).

\section{DNA sequence analysis of $S_{a}$ and $S_{b}$-RNase}

Based on the upstream and downstream primers designed against the adjacent exon region sequences, four intron PCR products of $S_{a}$ and $S_{b}$-RNase genes were obtained. The partial sequencing results at both ends of the fragments were consistent with the known sequences, so the resulting PCR products were the intended target segments. The first introns of $S_{a}$ and $S_{b}$-RNase were $\sim 15 \mathrm{~kb}$ long, and the second introns of $S_{a}$ and $S_{b}$-RNase were $\sim 15 \mathrm{~kb}$ and $8 \mathrm{~kb}$ long, respectively (Fig. 2A); these are rather long introns, which are rare in plants. Sanger sequencing of the PCR product library combined with high-throughput sequencing yielded the reference sequences of the first intron of $S_{a}$-RNase, the first and second introns of $S_{b}$-RNase, and part of the second intron of $S_{a}$-RNase. In addition, we obtained the $S_{a}$-RNase promotor sequence from splicing the sequences of the unmapped reads ${ }^{45}$ (Supplementary Dataset S1). The DNA reference sequences of $S_{a^{-}}$and $S_{b}$-RNase were used as query sequences for comparison with the sequences of F. viridis style transcripts (Supplementary Dataset S2) using BLAST. The intron and promotor sequences were matched exactly by six sequences, among which two (lncRNA1 and lncRNA2) corresponded to the promotor region of $S_{a}$-RNase, and four (lncRNA3, IncRNA4, lncRNA5, and lncRNA6) corresponded to the first intron of $S_{b}$-RNase (Fig. 2B). The six sequences had short ORFs that did not correspond to a deduced protein sequence in the style protein library; by extension, they lacked proteincoding ability, which is consistent with the characteristics of lncRNAs. In addition, some of lncRNAs have promotor structures, or located in the promotor structure region of S-RNase (Supplementary Table S4, Supplementary Table S5). Using the DNA sequences matched with IncRNA1 and 2 to query raw data and mapping the reads to the DNA regions showed that the two lncRNAs were from different transcripts (Supplementary Fig. S7). However, the four other lncRNAs (LncRNA3, lncRNA4, lncRNA5, and IncRNA6) could not be confirmed as four independent transcripts because their corresponding DNA sequences partially overlapped. Taking the different introns of lncRNA5 and lncRNA6 as clues, these four lncRNAs may have come from the alternative splicing of the same 


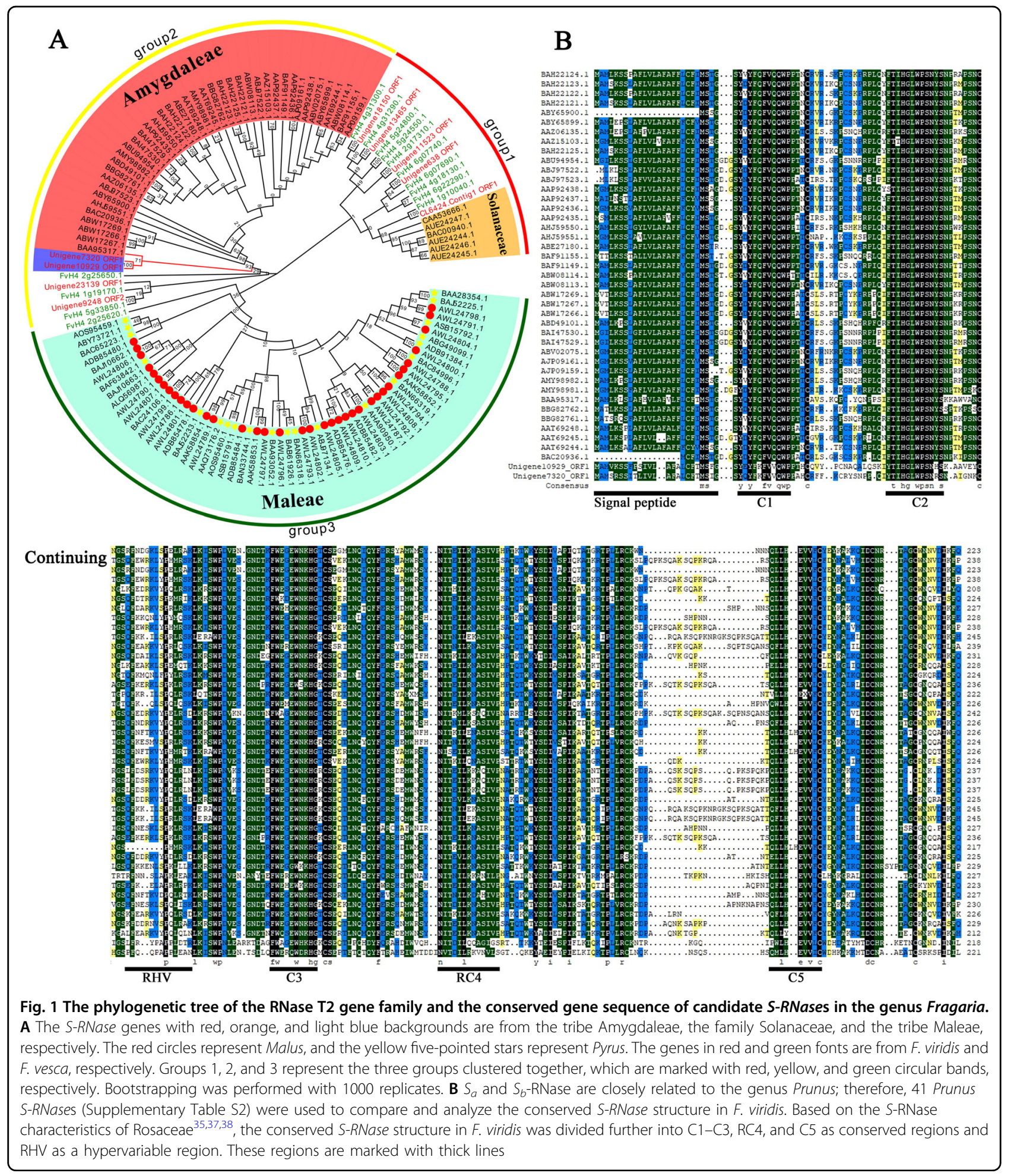

transcript (Supplementary Fig. S7). The sequences of lncRNA1, lncRNA2, lncRNA3, lncRNA4, lncRNA5, and IncRNA6 can be found in Supplementary Dataset S2 (sequence IDs, Unigene18323_All, Unigene16020_All,
Unigene26368_All, Unigene9260_All, Unigene25450_All, and Unigene12807_All, respectively). The DNA reference sequences of $S_{a^{-}}$and $S_{b}$-RNase are provided as Supplementary Sequence S1 and Supplementary Sequence S2. 
A

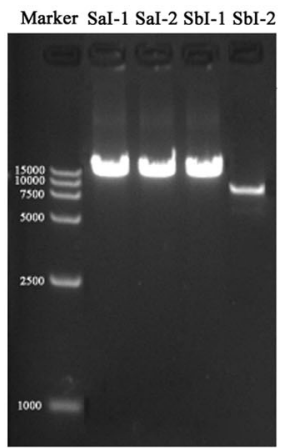

B
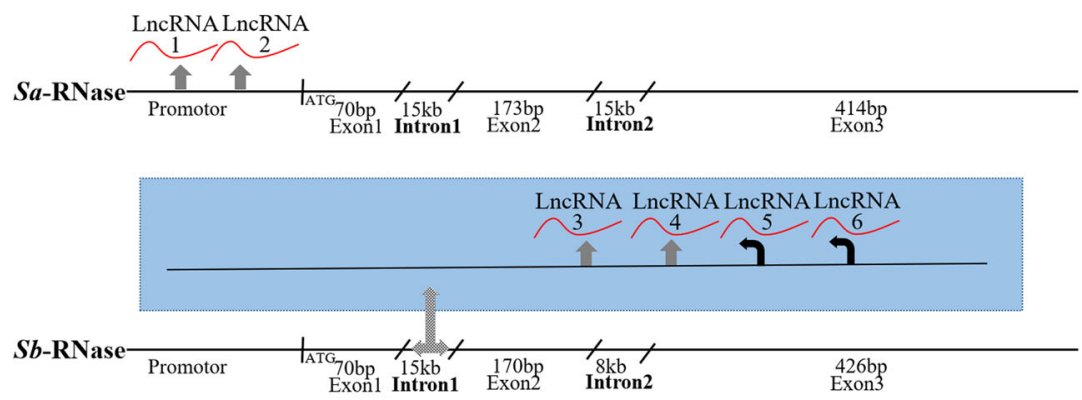

Fig. 2 The structural pattern and potential neighboring IncRNAs of S-RNase. A $S_{a} l-1$ and $S_{a} l-2$ represent the first and second introns of $S_{a}-R N a s e$, respectively; $S_{b} l-1$ and $S_{b} l-2$ represent the first and second introns of $S_{b}-R N a s e$, respectively. The purified PCR product was used for DNA agarose gel electrophoresis. A 15-kb marker is provided on the left as a reference. B The area between the back slashes on the gene represents introns. The first intron of $S_{b}$-RNase is magnified by a grid-filled arrow and highlighted with a blue background. The thick black line with arrow indicates the position and transcription direction of IncRNA. The analysis of the transcription direction of IncRNAs according to the characteristic structure of intron boundaries is also shown. The transcription direction could not be determined because there was no intron structure in IncRNA1-4, and the thick gray lines with arrows represent only the transcription positions
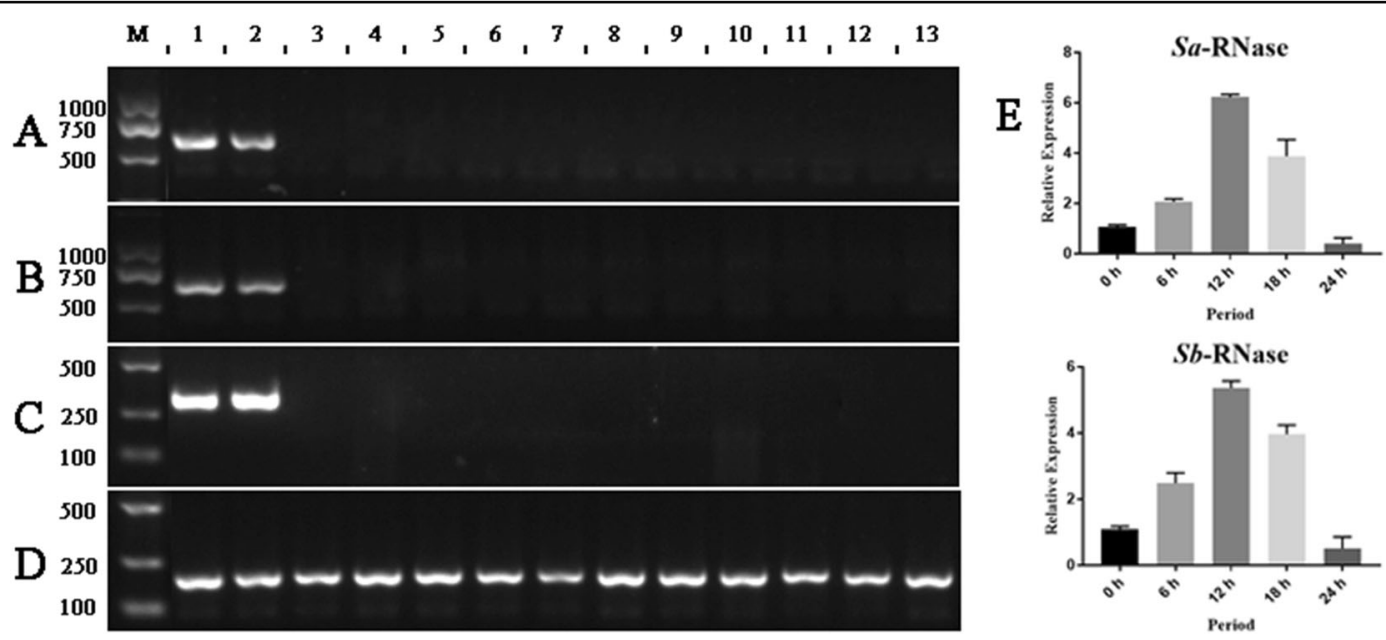

Fig. 3 Analysis of $\mathbf{S}$-RNase expression by RT and qRT-PCR. Lanes 1, 2, 3, 4, 5, 6, 7, 8, and 9 in $\mathbf{A}$-D represent the flower balls, styles, ovaries, receptacles, pedicels, calyxes, petals, leaves, and anthers of $F$. viridis 42 , respectively. Lanes 10, 11, 12, and 13 represent the flower balls of $F$. vesca $41, F$. mandshurica 43, F. nilgerrensis 45, and F.x ananassa "Benihoppe", respectively. A, B represent the expression of $S_{a}$ and $S_{b}$-RNase based on specific primers. C Shows the expression results obtained using degenerate primers for $S_{a}$ and $S_{b}$-RNase. D Represents the detection results of all cDNA samples with primers for a reference gene, EF-1a. E Illustrates the expression trends of S-RNase at different periods in styles after pollination. The abscissa represents the time after pollination, and the ordinate represents the level of expression

\section{Expression analysis of S-RNase in Fragaria tissues}

We analyzed the expression of S-RNase in styles, ovaries, receptacles, pedicels, calyxes, petals, leaves, and anthers. $S_{a^{-}}$and $S_{b}$-RNase are specifically expressed in the styles and conform to the expression principles of style determinants; this also explains why the flower balls containing styles can be used for the screening and expression analyses of $S$-RNase. To test the expression of $S$-RNase in the different germplasms, the incompatible $F$. viridis 42 was selected as the control, while F. vesca 41,
F. mandshurica 43, F. nilgerrensis 45 , and $F$. xananassa "Benihoppe" were selected as the compatible species for analyses using specific and degenerate primers $\left(\mathrm{FS}_{\mathrm{a}} \mathrm{S}_{\mathrm{b}}\right.$ and $\mathrm{RS}_{\mathrm{a}} \mathrm{S}_{\mathrm{b}}$ ) of $S$-RNase ( $S_{a}$ and $S_{b}$-RNase), and the cDNA of the flower balls was used as a template. The results showed that $S_{a}$ and $S_{b}$-RNase were expressed only in $F$. viridis 42 , and their expression was not detected in all compatible strawberry germplasms (Fig. 3A-D). The flower balls containing styles after pollination were used to test the spatiotemporal expression of the two genes at 
$6,12,18$, and $24 \mathrm{~h}$, and unpollinated flower balls were used as controls (Fig. 3E). The results showed that $S_{a^{-}}$and $S_{b}$-RNase expression first increased and then decreased after pollination, which was consistent with the degree of pollen tube inhibition ${ }^{45}$, and the highest levels of expression were achieved $12 \mathrm{~h}$ after self-pollination.

\section{$S$ locus mapping in Fragaria}

The deciphering of plant genomes and the construction of databases has made data mining related to agronomic traits increasingly convenient ${ }^{46,47}$. Consistent with the results of previous studies ${ }^{48,49}$, we noted the high genomewide collinearity of Fragaria with Rosa and Prunus (Supplementary Fig. S8), which provided favorable conditions for $S$ locus location in Fragaria. The $S$ loci were reported to be located on chromosomes 3 and 6 in the genomes of Rosa and Prunus, respectively ${ }^{16,49}$. We further analyzed the collinearity of Rosa (rose) chromosome 3 and Prunus (almond) genome chromosome 6 with Fragaria (F. vesca) and observed that both rose chromosome 3 and almond genome chromosome 6 had large collinear blocks with $F$. vesca chromosomes 1 and 6. However, further analysis revealed that the $S$ loci of rose and almond had a common collinear region with $F$. vesca chromosome 6 . In contrast, it had no collinear region with $F$. vesca chromosome 1 (Supplementary Fig. S9). The results indicated that there was only one $S$ locus on chromosome 6 in Fragaria and that there was no $S$ locus linked to SI on chromosome 1. We intercepted $2.5 \mathrm{Mb}$ of $S$-RNase flanking the rose and almond genomes as the corresponding $S$ loci regions and analyzed the collinearity relationship between them and the $F$. vesca genome. The results showed that the $F$. vesca genome blocks that were collinear with the $S$ loci regions of the Rosa_v1 rose genome, the Rosa_v2 rose genome, and the almond genome were 2.4 (Chr: 2792689-6206471), 3.4 (Chr: 2072839-5489398), and 7.0 (Chr: 3802-7052976) Mb, respectively (Fig. 4). The overlapping area was $2.7 \mathrm{Mb}$ (Chr: 2792689-5489398), which was located within 7.01-13.80\% of the chromosome length, and this area was regarded as the $S$ locus area in $F$. vesca. In addition, through wholegenome analysis of the gene families in $F$. vesca, we found two RNase T2 family genes (FvH4_6g07740, FvH4_6g07690) in the predicted $S$ locus region. Notably, there were F-box gene clusters near the two RNase T2 family genes (Fig. 4, Supplementary Fig. S2), which was consistent with the linkage characteristics of the $S$ locus genes (style and pollen determinants). However, these two RNase T2 family genes were not $S$-RNase genes (see the sections "S-RNase prediction in F. viridis and $F$. vesca" and "Evolutionary analysis of RNase T2 family members").

\section{Identification of the S-RNase genotype of $F$. viridis 42}

Polyacrylamide gel electrophoresis (PAGE) was used to identify nucleotide differences between the two genes, while genomic DNA was used as a template. Similar to that in Pyrus plants ${ }^{15}$, the SI of F. viridis is not complete, and a small number of progenies are derived from seeds contained in malformed fruits through self-pollination. First, a total of 29 lines, including wild $F$. viridis 42 (zero generation), selfed first generations, selfed second generations, and selfed third generations, were screened for the $S_{a}$ - and $S_{b}$-RNase genotypes using degenerate primers (Table 2 and Fig. 5). The detection results were consistent with those obtained using CDS full-length specific primers and style cDNA as the template (Supplementary Fig. S10). There were six lines containing $S_{a}$-RNase exclusively, 13 lines containing $S_{b}$-RNase exclusively, and 10 lines containing both $S_{a}$-RNase and $S_{b}$-RNase. There were only three genotypes $\left(\mathrm{S}_{\mathrm{a}} \mathrm{S}_{\mathrm{a}}, \mathrm{S}_{\mathrm{a}} \mathrm{S}_{\mathrm{b}}\right.$, and $\left.\mathrm{S}_{\mathrm{b}} \mathrm{S}_{\mathrm{b}}\right)$ without the $S_{a^{-}}$ and $S_{b}$-RNase null gene locus lines; therefore, we preliminarily considered $S_{a^{-}}$and $S_{b}$-RNase as alleles. To further confirm that $S_{a^{-}}$and $S_{b^{-}}$RNase were alleles, we selected "S1-02-S2-76-S3-09" $\left(\mathrm{S}_{\mathrm{a}} \mathrm{S}_{\mathrm{b}}\right)$ for self-crossing and obtained 214 progeny lines. The genotype was identified by $\mathrm{FS}_{\mathrm{a}} \mathrm{S}_{\mathrm{b}}$ and $\mathrm{RS}_{\mathrm{a}} \mathrm{S}_{\mathrm{b}}$. The genotype ratio was $\mathrm{S}_{\mathrm{a}} \mathrm{S}_{\mathrm{a}}: \mathrm{S}_{\mathrm{a}} \mathrm{S}_{\mathrm{b}}$ : $\mathrm{S}_{\mathrm{b}} \mathrm{S}_{\mathrm{b}}=48: 106: 60 \approx 1: 2: 1$. The progeny genotypes of "S102-S2-30-S3-09" $\left(\mathrm{S}_{\mathrm{a}} \mathrm{S}_{\mathrm{a}}\right)$ following selfing were all $\mathrm{S}_{\mathrm{a}} \mathrm{S}_{\mathrm{a}}$, and the progeny genotypes of "S1-02-S2-76-S3-11" $\left(\mathrm{S}_{\mathrm{b}} \mathrm{S}_{\mathrm{b}}\right)$ following selfing were all $\mathrm{S}_{\mathrm{b}} \mathrm{S}_{\mathrm{b}}$. The progenies of "S1-02S2-30-S3-09" $\left(\mathrm{S}_{\mathrm{a}} \mathrm{S}_{\mathrm{a}}\right)$ and "S1-02-S2-76-S3-11" $\left(\mathrm{S}_{\mathrm{b}} \mathrm{S}_{\mathrm{b}}\right)$ crosses were all $\mathrm{S}_{\mathrm{a}} \mathrm{S}_{\mathrm{b}} . S_{a^{-}}$and $S_{b}$-RNase conformed to the Mendel's law of segregation, which confirmed that $S_{a}$ - and $S_{b}$-RNase were alleles.

\section{Intraspecific hybridization between different $\mathrm{S}$ genotype lines of $F$. viridis}

To verify the functions of $S_{a^{-}}$and $S_{b}$-RNase in $F$. viridis SI, three genotypes, $S_{a} S_{a}, S_{b} S_{b}$, and $S_{a} S_{b}$, were selected to conduct selfing and intraspecific hybridization (Fig. 6, Supplementary Table S6). Each line was self-crossed, different lines of the same genotype were crossed with each other, and $S_{a} S_{b}$ genotype lines were used as the female parent in crosses with the $S_{a} S_{a}$ or $S_{b} S_{b}$ genotype lines. The hybridizations yielded abnormally developed receptacles with no fruit or malformed fruit, had low fruitset and seed-set rates in single fruits, and were of the incompatible type. The $\mathrm{S}_{\mathrm{a}} \mathrm{S}_{\mathrm{a}}$ and $\mathrm{S}_{\mathrm{b}} \mathrm{S}_{\mathrm{b}}$ genotype lines were used as the female parent in crosses with the $S_{a} S_{b}$ genotype line. Intercrossing between $S_{a} S_{a}$ and $S_{b} S_{b}$ genotype lines yielded normally developed receptacles, a high seedset rate, a fruit-set rate of $100 \%$, no abnormalities, and a compatible type. In the incompatible hybridizations, most of the pollen tubes stopped at $2 / 3$ of the length of the style $48 \mathrm{~h}$ after pollination (Fig. 7). In the compatible hybridizations, most pollen tubes extended to the base of the style, and some pollen tubes passed smoothly through the base of the style (Fig. 7). In addition, the color and development status of compatible and incompatible 


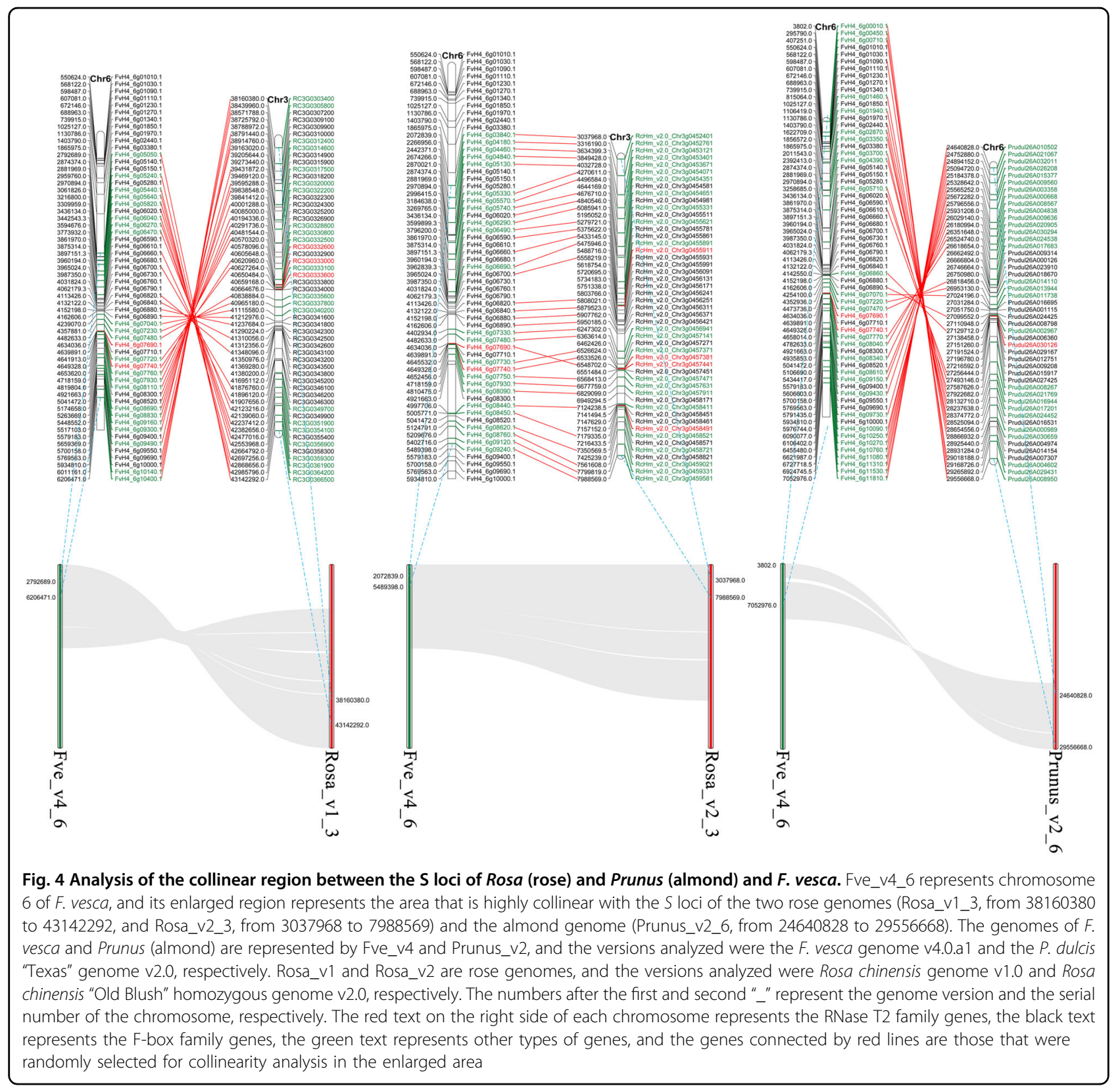

styles, receptacles, and achenes were different within $10 \mathrm{~d}$ of pollination (Supplementary Fig. S11). In summary, it has been confirmed that $S_{a^{-}}$and $S_{b}$-RNase are determinants of style SI in F. viridis.

\section{Interspecific hybridization between $F$. viridis, $F$. vesca, $F$. nilgerrensis, and $F$. mandshurica}

$F$. vesca 41, F. mandshurica 43 , and F. nilgerrensis 45 can produce numerous seeds after self-pollination, with the receptacle developing normally, a fruit-set rate of $100 \%$, and no malformed fruit; they, therefore, exhibit compatibility (Fig. 8, Supplementary Table S7). However, controversy persists about the (in)compatibility of
F. mandshurica ${ }^{29,30,50}$, which was observed to be a selfcompatible species according to our experimental data. When $F$. viridis 42, as the male parent, was crossed with $F$. vesca $41, F$. mandshurica 43 , and $F$. nilgerrensis 45 , the single-fruit seed-set rates of $F$. vesca 41 and $F$. nilgerrensis 45 were high, the receptacles grew and expanded, and the crosses exhibited compatibility; these results are consistent with previous reports ${ }^{1,5}$. However, $F$. nilgerrensis 45 exhibited a less-developed receptacle, and the seed-set rate was significantly lower than that obtained from selfing. F. mandshurica 43 hardly set seeds, and the receptacle was not developed, which indicated incompatibility. $F$. viridis 42 was crossed as the female parent with $F$. vesca 


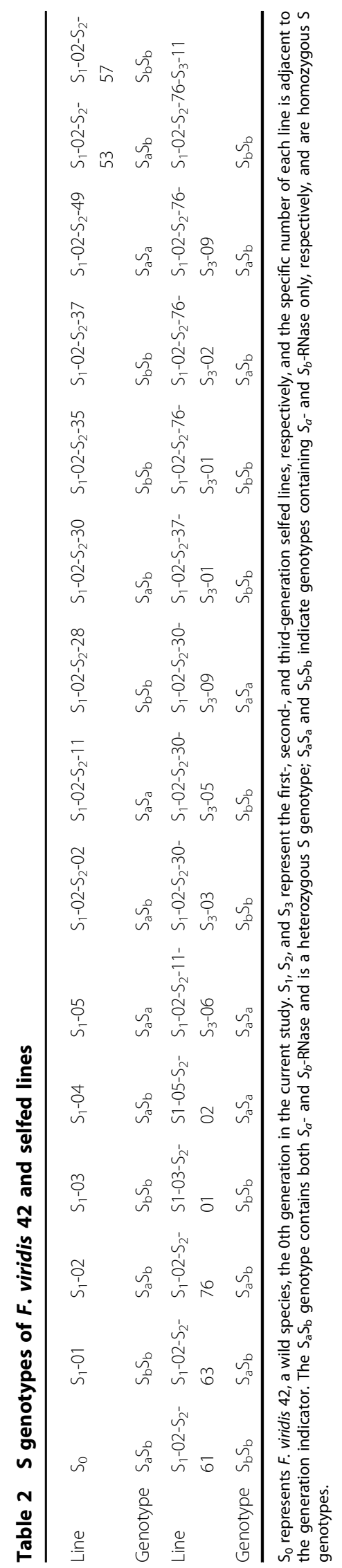

41 and F. nilgerrensis 45 . The results showed no receptacle development and almost no achene, which are considered signs of incompatibility. These results are consistent with the findings of the previous studies ${ }^{1}$. When $F$. viridis 42 was crossed as the female parent with F. mandshurica 43, receptacles developed normally, many achenes were produced, the fruit-set rate was $100 \%$, and there was no malformed fruit. These results indicated compatible interspecific hybridization. When $F$. vesca 41 was crossed as the female parent with $F$. nilgerrensis 45 and $F$. mandshurica 43 or when $F$. vesca 41 was crossed as the male parent with $F$. mandshurica 43 , the developed receptacles were normal, and the seed-set rate was high. These results indicate that these are compatible interspecific hybridizations. However, when $F$. vesca 41 , as the male parent, was crossed with $F$. nilgerrensis 45 , flower receptacle development and seed-set rate were not superior to those observed under $F$. nilgerrensis 45 selfpollination. When $F$. mandshurica 43 was crossed as the male parent with $F$. nilgerrensis 45 , the receptacles enlarged and had a certain seed-set rate, which was also considered a sign of compatible hybridization. However, the values from this hybridization were considerably lower than those obtained from $F$. nilgerrensis 45 selfing. In the reverse crossing, there were almost no seeds, and the receptacle did not develop. These results indicate an incompatible hybridization.

The growth status of the pollen tubes in the styles was investigated at $48 \mathrm{~h}$ after self- and cross-pollination, and the results are shown in Fig. 8. In contrast to the incompatible case in $F$. viridis 42 , the pollen tubes of $F$. vesca 41, F. mandshurica 43 , and $F$. nilgerrensis 45 smoothly extended through the base of the style after self-pollination. Compatible interspecific hybridization (F. vesca 41, as the female parent, crossed with $F$. viridis 42, F. mandshurica 43, and F. nilgerrensis 45; F. viridis 42, as the female parent, crossed with $F$. mandshurica 43; $F$. nilgerrensis 45 , and $F$. mandshurica 43 , as the female parent, crossed with $F$. vesca 41) was found to be characterized by pollen tube growth patterns similar to those observed after the self-pollination of compatible germplasms. However, when F. nilgerrensis 45 , as the female parent, was crossed with $F$. viridis 42 and $F$. mandshurica 43 , the growth of most pollen tubes was restrained at 1 / $3-1 / 2$ of the length of the style, and only a small number of uninhibited pollen tubes extended through the bases of the partial styles. In the incompatible interspecific hybridizations-when $F$. mandshurica 43 (female parent) was crossed with $F$. viridis 42 and $F$. viridis 42 (female parent) was crossed with $F$. vesca 41 -the growth of most pollen tubes was restricted at $1 / 3-1 / 2$ of the length of the style. In addition, when $F$. viridis 42 and $F$. mandshurica 43 (female parents) were crossed with $F$. nilgerrensis 45 , the growth of most germinated pollen tubes was considerably 

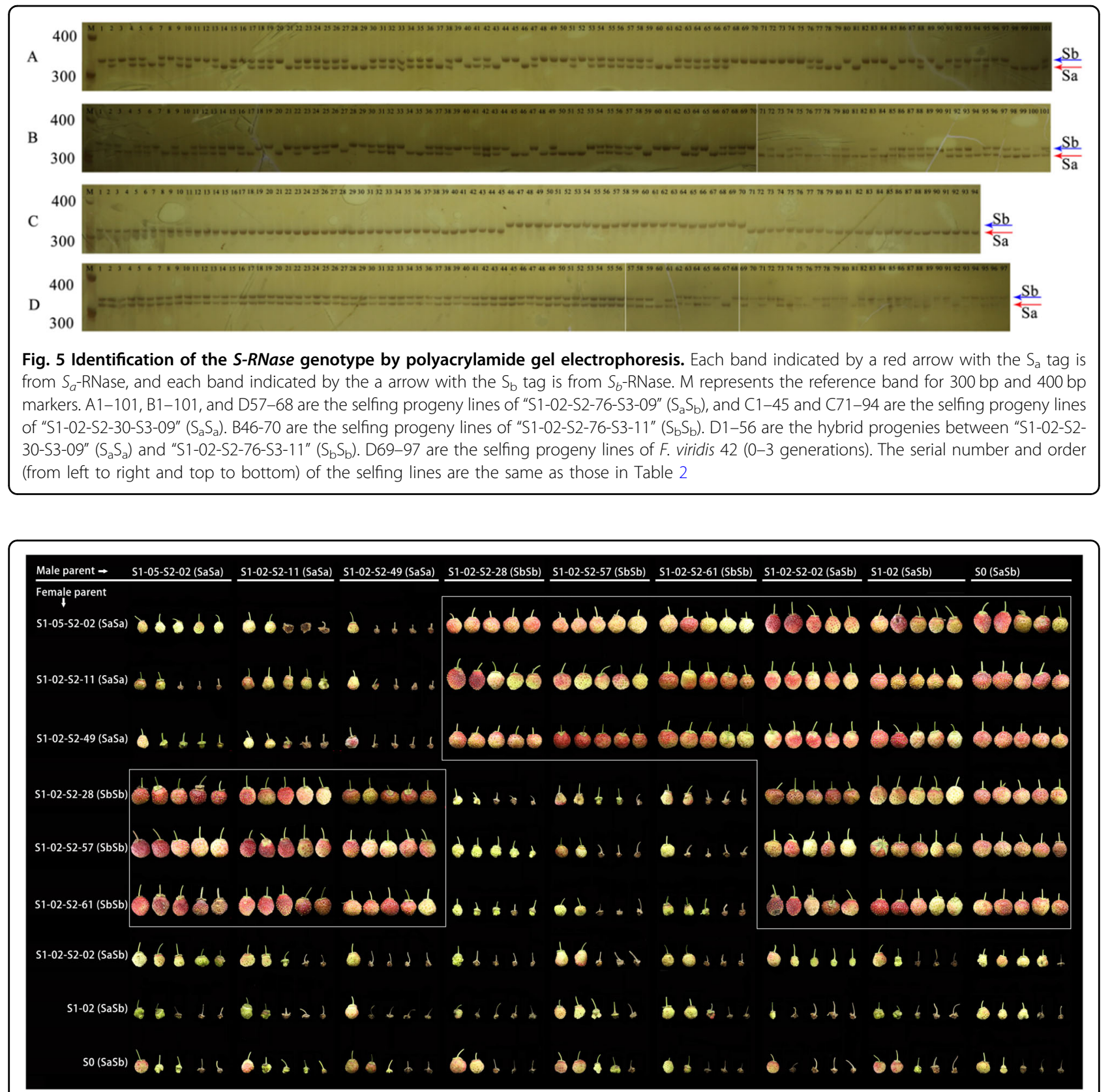

Fig. 6 Fruit development following hybridization of different genotype lines from $\boldsymbol{F}$. viridis $\mathbf{4 2}$. The $\mathrm{S}$ genotype lines were selected as shown in Table 2. The S-RNase genotype is shown in brackets

inhibited, reaching only $1 / 5$ of the length of the style. We also observed that pollen from $F$. nilgerrensis 45 exhibited lower germination rates than that from $F$. vesca 41 and F. viridis 42 on the stigma of $F$. mandshurica 43 and that the pollen tubes did not pass easily through the stigma.

\section{Discussion}

S-RNase-based GSI in the genus Fragaria

Relatively extensive research has been carried out on SI in the subfamily Amygdaloideae (apricot, Japanese apricot, plum, etc.) and the tribe Maleae (apple and pear ${ }^{17}$. Strawberry plants belong to another subfamily, Rosoideae, in the family Rosaceae, and the mechanism of the regulation of incompatibility in this subfamily remains unclear. Based on the results of $S$ genotype identification and cross-pollination experiments, $S_{a^{-}}$and $S_{b}$-RNase are determinants of SI in the styles of $F$. viridis, and $S_{a^{-}}$and $S_{b}$-RNase are alleles of the $S$ locus. Furthermore, the genus Fragaria has an $S$-RNase-based SI type, which demonstrates the unproven GSI mechanism in Rosoideae 


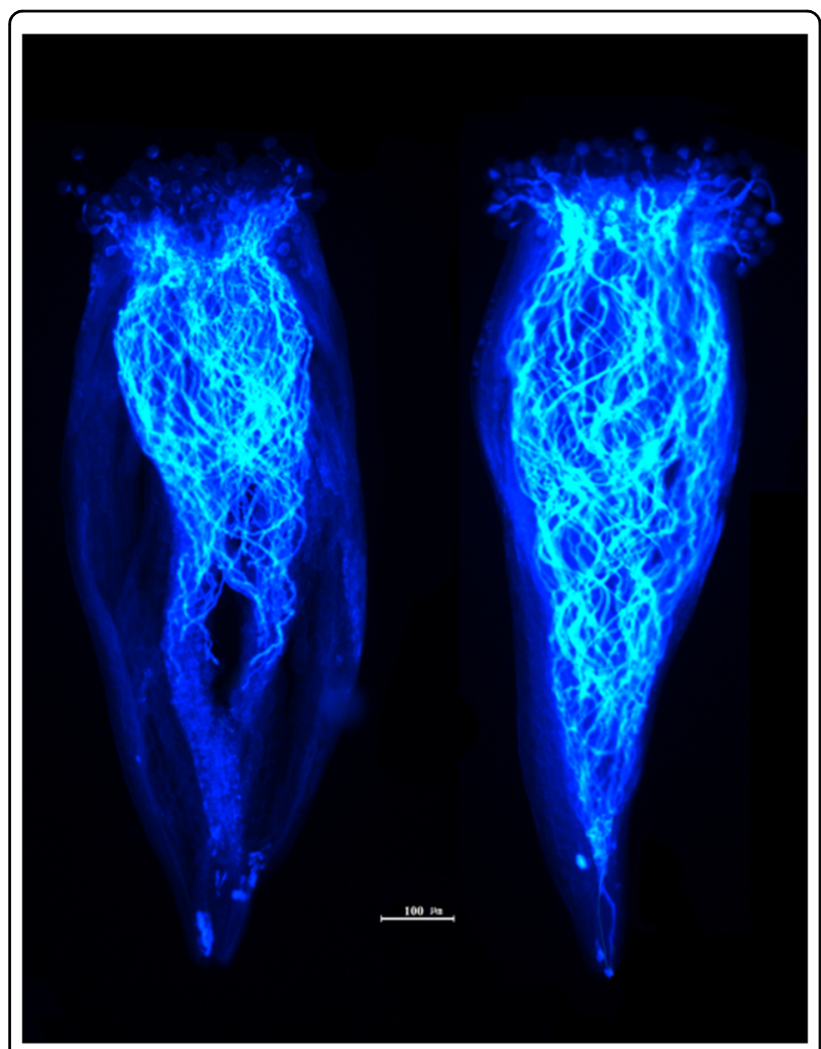

Fig. 7 Growth status of compatible and incompatible pollen tubes in the styles of $F$. viridis lines $48 \mathrm{~h}$ after pollination. Scale bar $=100 \mu \mathrm{m}$. The left side shows pollen tube growth in the incompatible state, that is, the hybridizations that are not highlighted in "Fig. 6". The right side shows pollen tube growth in the compatible state; that is, the hybridizations that are shown in boxes in "Fig. 6"

and further advances our understanding of the SI system in Rosaceae. These observations are also in agreement with a recently published paper identifying the S-locus in the genus $\operatorname{Ros}^{51}$. Most pollen tubes are generally suppressed at $1 / 3-1 / 2$ of the style from the stigma when SI occurs $^{10,15,52,53}$. Consistent with the results of previous studies $^{45}$, the SI of Fragaria plants responds relatively slowly to pollen after pollination, implying some differences in the intermediate mechanism or the SI modification factors.

Analysis of the SC mechanism in the genus Fragaria and insights into the S-RNase expression regulation mechanism

Notably, there are some self-compatible germplasms in the Rosaceae family with SI systems ${ }^{1,15,17,54}$. Such compatibility phenomena are relatively widespread in plants of the genus Fragaria, not only in cultivated species but also in many wild species, such as $F$. vesca, $F$. nilgerrensis, and F. mandshurica ${ }^{1,6,29}$. Consistent with previous reports, genome-wide RNase T2 family analysis has confirmed that no S-RNase has been observed in self-compatible species $^{6,18}$. Further clues about the compatibility of the germplasm in the genus Fragaria were found through collinearity analyses. Two RNase T2 family genes were present at the identified $S$ locus; however, the two genes did not conform to the characteristics of S-RNase. The conversion of a self-incompatible germplasm into a selfcompatible germplasm often occurs in Rosaceae, typically due to variations in the genome sequence that result in the loss of gene fragments or gene functions of $S$ determinants in the style or pollen ${ }^{20,21,23,55-58}$. Our results suggest that $S$-RNase was lost at the $S$ locus of selfcompatible germplasms during evolution.

$S$-RNase is expressed specifically in the styles and is maintained at higher levels after self-pollination, which is very important for the development of the SI phenotype $^{24,25}$. However, few reports have explored the regulation of S-RNase expression, and only the promotor of $S$-RNase has been identified ${ }^{59,60}$. The self-incompatible $S$ locus is located at the centromere (Solanaceae) or subtelomeric position on the chromosome. The region is often hypermethylated ${ }^{61}$, highly repetitive in its sequence, and heterochromatised ${ }^{62}$; these properties may make the DNA conservative and difficult to recombine and, in turn, restrict gene expression ${ }^{61,63,64}$. Fernández i Marti et al. ${ }^{27}$ showed that the methylation of the $S$ locus in almonds is related to the loss of $S$-RNase function. In addition, the roles of introns in expression regulation have also been increasingly reported ${ }^{26,29,65}$. S-RNase in the genus Fragaria has a large intron and is currently the longest $S$ gene; these characteristics are also rare in plant genes. However, whether such large introns are also involved in gene expression regulation requires further investigation. Some noncoding sequences can be expressed and play important roles in gene regulation ${ }^{66,67}$. Here, we detected the existence of related adjacent long noncoding RNAs near $S$-RNase, including the promotor and intron regions, which also provide further new clues for S-RNase expression regulation. All of these findings indicate the complexity of $S$-RNase expression regulation, which may involve multiple processes. However, much research work is still needed to clarify its mechanism.

\section{$\mathrm{UI}$ in Fragaria spp}

Generally, UI between SC and SI species arises when an SI species, as a pollen donor is crossed, with an SC species and is compatible, however the reciprocal cross occurs, and it is manifested as an incompatibility ${ }^{7}$. Among the three interspecific hybridizations between $F$. viridis and $F$. vesca, $F$. mandshurica, and $F$. nilgerrensis, two hybridizations conformed to the rule above and were consistent with previous research results, suggesting that the $\mathrm{UI}$ is related to the $S$-RNase-based $\mathrm{SI}^{1,6,68}$. However, when $F$. viridis was crossed with $F$. mandshurica, the result was contrary to the $\mathrm{SI} \times \mathrm{SC}$ rule and was not influenced by the 


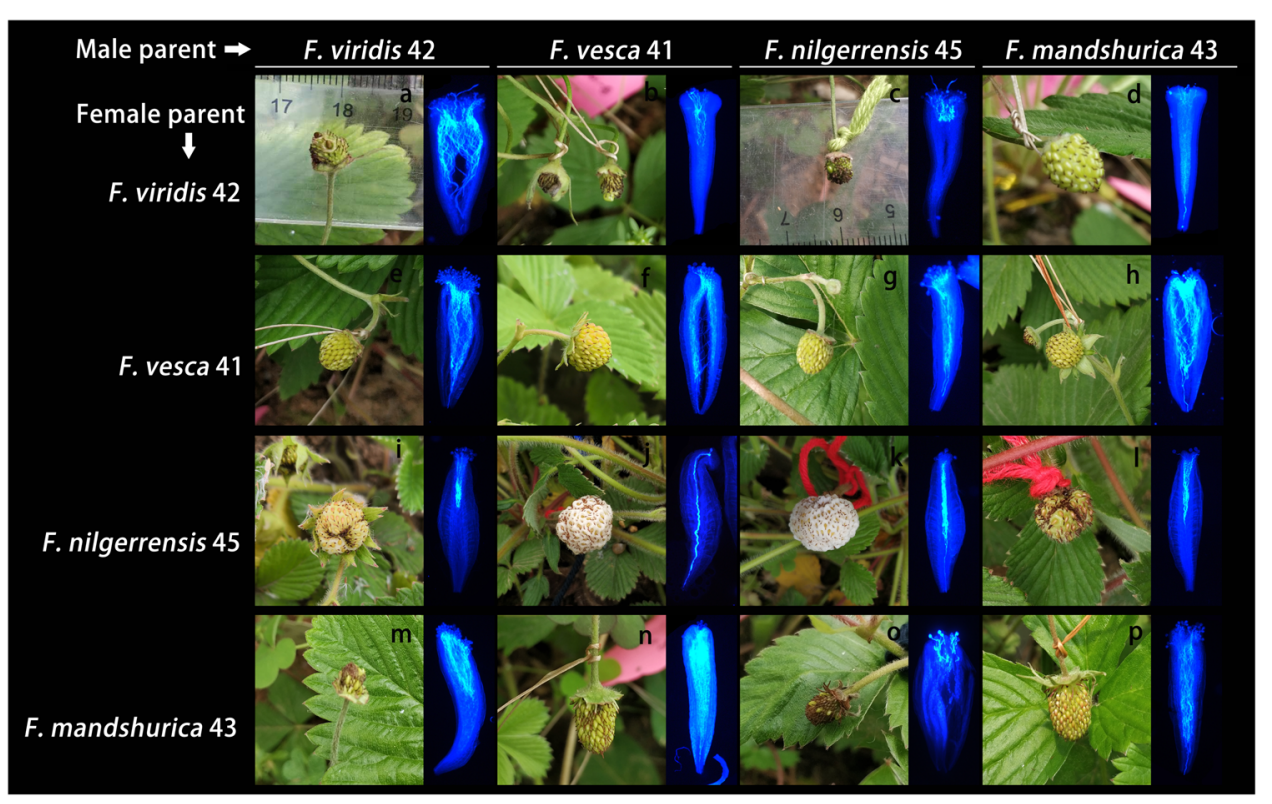

Fig. 8 Fruit development and pollen tube extension in the style after interspecific cross-pollination. $F$. viridis 42 is a self-incompatible species, and F. vesca 41, F. mandshurica 43, and F. nilgerrensis 45 are self-compatible species. All are wild germplasm resources. Pictures were obtained 15-25 days after pollination, and the pollen tube was selected for observation $48 \mathrm{~h}$ after pollination

S genotype (Fig. 8, Supplementary Table S7). Pollen rejection under interspecific hybridization systems is complex and involves multiple pathways. In addition to the UI that conformed to the SI $\times$ SC rule, there are other $\mathrm{UI}$ barriers that are not related to $\mathrm{SI}^{69,70}$. An SIindependent mechanism exists not only between compatible germplasms (such as F. nilgerrensis and F. mandshurica) but also between SI and SC germplasms (such as F. viridis and F. mandshurica) (Fig. 9). The discovery of an exception to the $\mathrm{SI} \times \mathrm{SC}$ rule that is in force in Fragaria and the results of the investigation of the UI between compatible species provide a basis for the analysis of Fragaria UI mechanisms.

In other genera, including Solanum and Pyrus, crosscompatible pollen tubes can reach the base of the style, and some even pass through the style bottom. However, interspecific incompatible pollen tubes often stop growing within $1 / 3$ of the length of the style $7,10,15,52$. In Solanum, interspecific incompatible pollen tubes are arrested earlier than self-incompatible pollen tubes ${ }^{7}$; a similar phenotype was also observed in Fragaria. There were also some differences in the inhibition time between different interspecific hybridizations in Fragaria plants. Some interincompatible pollen tubes $(1 / 3-1 / 2)$ in Fragaria are suppressed later than those in Solanaceae ${ }^{7}$ (within $1 / 3$ of the length of the style); however, some interspecific hybridizations are stopped earlier. Consistent with previous results, $F$. vesca accepts pollen from other strawberry species relatively easily ${ }^{1,5,31}$. In the present study, F. nilgerrensis had a lower capacity to accept pollen from

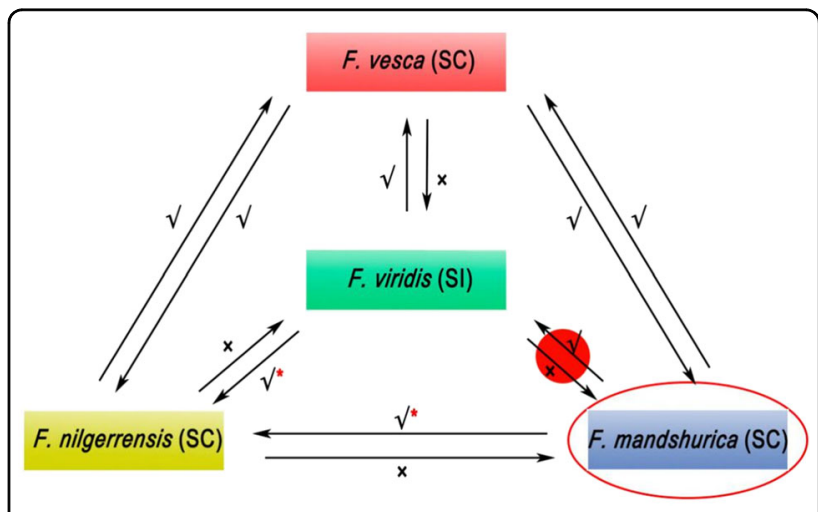

Fig. 9 Hybridization of four wild strawberry diploid germplasms. The arrows in the diagram point to the female parents. $\sqrt{ }$ denotes compatibility, $x$ denotes incompatibility, and $\sqrt{ }^{*}$ denotes

hybridizations that were considered compatible in this study. In fact, the $\sqrt{ }^{*}$ hybridizations are different from the other completely compatible interspecific hybridizations (that is, the receptacle develops normally, the number of achenes is high, and most germinated pollen tubes can reach the bases of styles), although a certain compatibility was observed based on receptacle development and achene level. In the $\sqrt{ }^{*}$ interspecific hybridizations, most pollen tubes were inhibited at $1 / 3-1 / 2$ the length of the style, and only some of the styles facilitated pollen tubes without restriction

germplasms other than $F$. vesca, and the intensity of pollen suppression was greater in the pistils of other germplasms. The functional structural units involved in incompatibility include the pistil-side barrier and pollenside resistance. Additionally, incompatibility occurs when pollen resistance is lacking or is weaker than the 
pistil-side barrier ${ }^{7,8,71}$. The interspecific hybridization barrier is also a relatively primitive state that prevents gene exchanges among related germplasms ${ }^{72}$. We speculate that when the pistil-side barrier in $F$. vesca weakened, the style of $F$. nilgerrensis maintained a certain capacity to overcome pollen resistance, and the pollen developed relatively weak resistance during evolution. These results explain why $F$. vesca is a reasonable candidate intermediate germplasm for gene exchange and why $F$. nilgerrensis 45 , in a compatible interspecific hybridization, hinders most of the pollen tubes of $F$. viridis and F. mandshurica. They also explain why the pollen tubes of $F$. nilgerrensis are strongly restricted in the styles of $F$. viridis and $F$. mandshurica in incompatible interspecific hybridizations. Moreover, the phylogenetic evolution of Fragaria shows that $F$. nilgerrens has a distant relationship with $F$. viridis and $F$. mandshurica ${ }^{73}$. Thus, the influence of phylogenetic distance on the compatibility of interspecific hybrids cannot be ruled out.

\section{The control loci of S-RNase-mediated GSI}

Bosković et al. ${ }^{6}$ hypothesized that two RNase loci (S and $\mathrm{T})$ can explain the regular occurrence of SI and UI in the genus Fragaria. However, this hypothesis-that SI is controlled by $S$-RNase-is constrained by the fact that not all style-expressed RNases are $S$-RNases ${ }^{18,74}$. Sequence information for these two loci has not been obtained. In addition, peptide sequences that have been previously used to demonstrate that the $\mathrm{S}$ and $\mathrm{T}$ loci encode RNase proteins are controversial ${ }^{6,18}$. In contrast to previous findings, only two allelic S-RNases could be identified using the deduced style protein database of $F$. viridis based on information regarding the characteristics of $S$-RNase, and the two genes were identified as style SI determinants using the hybridization experiment; these findings indicate that only one $S$ locus plays a role in SI in the genus Fragaria. Based on genome-wide collinearity analysis, the $S$ locus regions of the almond and rose genomes had only one collinear region with the $F$. vesca genome, on chromosome 6 (Chr6: 2792689-5489398). Pgl1 exists in this region (Supplementary Fig. S9), which is consistent with the previously reported T locus ${ }^{6}$. The $S$ locus predicted using collinearity analysis is also located at the far end of the chromosome, which is consistent with the subtelomeric region where the $S$ locus of the species is observed in families other than Solanaceae ${ }^{15,16,61}$. Bosković et al. $^{6}$ mentioned an $S$ locus linked to AC8 on chromosome 1; collinearity analysis also shows that chromosome 1 of the genus Fragaria has regions that are collinear with the chromosomes where the $S$ locus is found in almond and rose. However, the two pairs of collinear regions do not overlap in the Fragaria genome and are not located in the $S$ locus region in the genomes of almond and rose. The collinearity between the non-S locus chromosomal region of the chromosome where the $S$ loci of the Prunus and Rosa genomes are located and chromosome 1 of Fragaria might be the result of chromosomal exchange between chromosomes 1 and 6 of Fragaria $^{49,75}$. The AC8 genetic marker is located in the region that is collinear between Fragaria and Prunus (Supplementary Fig. S9) and may therefore reflect a false $S$ locus.

\section{Materials and methods}

\section{Materials and sample selection}

The incompatible wild $F$. viridis 42 was used to establish a style expression database and to explore style SI determinants in the genus Fragaria, and its selfed progenies were used for $\mathrm{S}$ genotype identification and functional verification by cross-pollination. To explore the reasons for the compatibility of certain strawberry germplasms, we selected $F$. vesca 41 (SC), F. nilgerrensis 45 (SC), F. mandshurica 43 (SC), and F.x ananassa "Benihoppe" (SC) for an analysis of the expression of $S$-RNase using RT-PCR. This was accompanied by cross-pollination experiments among four wild germplasms to further analyze the role played by $S$-RNase in the interspecific hybridization of the genus Fragaria. All resources used in the experiments were stored at the Baima Teaching and Research Base of Nanjing Agricultural University in Baima Town, Nanjing City, Jiangsu Province, China. Strawberry resources grown in the field (from March 2019 to June 2019) were used for selfing, cross-pollination, and sample collection. The temperature during this period was suitable for strawberry growth and enabled the plant materials to bloom normally. In addition, the flowering periods of the four Fragaria species partially overlapped. Various flowering stages differentially influence pollination results; therefore, we specifically selected flowers at the large bud stage (C3-C5) (Supplementary Fig. S12) for the pollination experiments.

\section{Total RNA extraction, cDNA preparation, and Illumina sequencing}

A total of six biological samples-three $F$. viridis flower balls (Supplementary Fig. S12) at 0 and $24 \mathrm{~h}$ after self-pollination-were harvested. Each sample included 10 flower balls, and all samples were stored in liquid nitrogen for RNA extraction. Total RNA was extracted using TRIzol reagent (TaKaRa, Dalian, China). RNA quality was evaluated using the Agilent 2100 RNA 6000 Kit. This RNA was then reverse transcribed into cDNA using SuperScript III Reverse Transcriptase (Invitrogen, CA, USA). Then, cDNA libraries were sequenced on an Illumina $\mathrm{HiSeq}^{\mathrm{TM}} 4000$ platform at the Beijing Genomics Institute (BGI, Shenzhen, China).

\section{Analysis of RNase T2 and F-box gene family members}

According to the hidden Markov model (HMM) of RNase T2 and the F-box domain, the hmmsearch 
program in Hmmer software (http://hmmer.org/ download.html) ${ }^{76}$ was used to search the genomic protein database of $F$. vesca and $F$. viridis to obtain the RNase $\mathrm{T} 2$ and F-box gene family members. To verify the integrity of the protein domains and filter out proteins that did not contain RNase T2 domains, the selected proteins were checked using the National Centre for Biotechnology Information (NCBI) Conserved Domains Database (https://www.ncbi.nlm.nih.gov/cdd/?term). The molecular weight and isoelectric point of the obtained RNase T2 family members were analyzed by ExPaSy (https:// web.expasy.org/protparam $/)^{77}$, and the signal peptides were analyzed by SignalP (http://www.cbs.dtu.dk/ services/SignalP/index.php $)^{78}$. Meanwhile, alignment analysis of the amino acid sequence was performed using DNAMAN8.0 (Lynnon, QC, Canada).

The RNase T2 HMM can be obtained via two methods. One is by downloading the RNase T2 model file (PF00445, RNase T2 HMM2) directly from the Pfam database (http://pfam.xfam.org/), and the other is by constructing an RNase T2 HMM using a known $S$-RNase (Supplementary Table S2). The $S$-RNases of Solanaceae, Malus, Pyrus, and Prunus were downloaded by entering the respective keywords (family or genus name and $S$-RNase) in the search window of the NCBI database. According to the annotations, some genes with unclear functions or incomplete sequences were omitted from the analysis, and the more reliable $S$-RNase genes, which were recognized $S$-RNase family members (Supplementary Table S2), were retained. The F-box HMM model file (PF00646, F-box HMM) was obtained directly from the Pfam database.

The proteome data for $F$. vesca were downloaded from the Genome Database for Rosaceae (https://www. rosaceae.org/), and the $F$. vesca genome $\mathrm{v}$ 4.0.a1 was used. The $F$. viridis style proteome was predicted based on the style transcriptome (Supplementary Dataset S2). The ORF finder script ${ }^{79}$ was used to predict the open reading frames (ORFs) (Supplementary Dataset S3) of the spliced transcripts and to deduce the corresponding protein sequences (Supplementary Dataset S4). De novo assembly was performed using clean reads from raw data with Trinity software ${ }^{80}$ for the transcripts. Afterward, the transcripts of all samples were merged (Supplementary Dataset S2).

The S-RNase in Supplementary Table S2 and the RNase T2 family members with longer protein lengths $(>100$ amino acids) from $F$. vesca and $F$. viridis were subjected to multiple sequence alignment using the MUSCLE program in MEGA $7.0^{81}$. A phylogenetic tree was constructed using MEGA 7.0, while the neighbor-joining method was adopted for cluster analysis, with the number of bootstrap replicates set to 1000. EvolView v2 (https://evolgenius.info//evolview$\mathrm{v} 2 / \# \operatorname{login}$ ) was used to improve the display of the evolutionary tree as well as to add group labels and colors.
According to the results of the evolutionary analysis, the species closely related to the candidate $S$-RNases of $F$. viridis were selected in order to analyze the conservative structure of the candidates.

\section{Cloning and sequence analysis of Sa-RNase and Sb-RNase}

RNA was extracted from the flower balls containing the styles of $F$. viridis 42 (Supplementary Fig. S12) using an RNAprep Pure Plant Plus Kit (TIANGEN, Beijing, China) according to the manufacturer's instructions. The kit is suitable for Rosaceae plants, which have tissues with high polysaccharide and polyphenolic compound contents. The extracted total RNA was digested with RNase-free DNase (TaKaRa, Dalian, China) to obtain high-purity RNA, and then the concentration was measured using a NanoDrop 2000 ultraviolet-visible spectrophotometer (Thermo Scientific, Waltham, MA, USA). The PrimeScript $^{\mathrm{TM}}$ RT Reagent Kit (TaKaRa, Dalian, China) was used to reverse transcribe the RNA into cDNA, according to the manufacturer's instructions. After reverse transcription, the appropriate amount of $\mathrm{dd}_{2} \mathrm{O}$ was added to dilute the mixture to $200 \mathrm{ng} / \mu \mathrm{L}$ based on the desired concentration. Specific primers were designed (Supplementary Table S3), including $\mathrm{FS}_{\mathrm{a}} \mathrm{CDS}$ and $\mathrm{RS}_{\mathrm{a}} \mathrm{CDS}$ for $S_{a^{-}}$ RNase and $\mathrm{FS}_{\mathrm{b}} \mathrm{CDS}$ and $\mathrm{RS}_{\mathrm{b}} \mathrm{CDS}$ for $S_{b}$-RNase, with cDNAs as templates for the CDS full-length sequences. PrimeSTAR ${ }^{\circledR}$ GXL DNA Polymerase (TaKaRa, Dalian, China) was used in the reaction. The amplification procedure for the $S_{a}$-RNase was as follows: 1 cycle $\left(98^{\circ} \mathrm{C}\right.$ for $5 \mathrm{~min}$ ), 36 cycles $\left(98^{\circ} \mathrm{C}\right.$ for $10 \mathrm{~s}, 50^{\circ} \mathrm{C}$ for $15 \mathrm{~s}$, and $68^{\circ} \mathrm{C}$ for $40 \mathrm{~s}$ ), a final extension at $68^{\circ} \mathrm{C}$ for $10 \mathrm{~min}$, and storage at $10^{\circ} \mathrm{C}$. The annealing temperature of $S_{b}$-RNase was $53{ }^{\circ} \mathrm{C}$. The temperature conditions for the other reactions were similar to those described above. The PCR products obtained after the addition of a base (A) at both ends of the obtained flat-end sequences were analyzed on $1.5 \%$ agarose gels, and the putative fragments were purified using a DNA purification kit (TaKaRa, Dalian, China). The procedure for adding the " $\mathrm{A}$ " base was conducted at $72{ }^{\circ} \mathrm{C}$ for 30 min using rTaq (TaKaRa, Dalian, China). The purified fragment was cloned into the pMDTM19-T vector (TaKaRa, Dalian, China) and transformed into E. coli $\mathrm{DH} 5 \alpha$ competent cells (Tsingke, Nanjing, China). Ten monoclones per gene were sequenced by a biotechnology company (Tsingke, Nanjing, China). The sequence obtained was compared with the sequence from the transcriptome using DNAMAN v8.0 (Lynnon, QC, Canada).

The target PCR product could not be obtained from the genome using cloning primers designed with the fulllength CDS of S-RNase. This indicated that S-RNase may contain large introns. To obtain genetic information regarding $S$-RNase, the nucleotide sequences of S-RNase were first analyzed to identify the exon-intron structure of 
the gene. Then, specific primers against the intron were designed at the intron-exon boundary of the gene using the segmented cloning approach. The GXL enzyme was employed to amplify the target fragment using a rapid reaction program. The annealing temperature was $60^{\circ} \mathrm{C}$, and the extension time was $1 \mathrm{~min}$ and $50 \mathrm{~s}$. Fresh young leaves of $F$. viridis were collected and stored at $-80^{\circ} \mathrm{C}$ in an ultralow-temperature refrigerator after quick freezing in liquid nitrogen. gDNA was extracted using the Super Plant Genomic DNA Kit (TIANGEN, Beijing, China), which is suitable for plants with high polysaccharide and polyphenolic contents. The following steps were used to design primers for S-RNase cloning: (1) the quality of the original reads (paired-end sequences) obtained from whole-genome resequencing data ${ }^{45}$ of Ls-S2-53 was evaluated and filtered to obtain clean reads; (2). SOAPdenovo $2^{82}$ was used to assemble clean reads that were not mapped to the reference genome, and a series of contigs and scaffolds were obtained as the database sequences; (3) $S_{a}$ and $S_{b}$-RNase transcripts representing query sequences were aligned with database sequences, and the boundaries of introns and exons were defined by analyzing the alignment results between $S$-RNase and its matched genome splicing sequence; and 4) after clarifying the number and positions of introns, upstream and downstream primers were designed against the first exon and the second exon or their matching sequences (contigs and scaffolds) and were used to clone the first intron; additional primers were designed against the second exon and the third exon or their matching sequences in order to clone the second intron. The primers used to clone the first and second introns of $S_{a}$-RNase were $\mathrm{FS}_{\mathrm{a}}$ Intron1 and $\mathrm{RS}_{\mathrm{a}}$ Intron1 and $\mathrm{FS}_{\mathrm{a}}$ Intron2 and $\mathrm{RS}_{\mathrm{a}}$ Intron2, respectively. The primers used to clone the first intron of $S_{b}$-RNase were $\mathrm{FS}_{\mathrm{b}}$ Intron 1 and $\mathrm{RS}_{\mathrm{b}}$ Intron1, and those used to clone the second intron were $\mathrm{FS}_{\mathrm{b}}$ Intron2 and $\mathrm{RS}_{\mathrm{b}}$ Intron2. The primer sequences are listed in Supplementary Table S3.

\section{F. viridis $\mathrm{S}$ genotype identification}

Using specific primers against the CDS (full-length) of $S_{a^{-}}$and $S_{b^{-}}$RNase and style cDNA after $12 \mathrm{~h}$ of selfpollination (template), the genotypes of the materials were determined (Table 2). The $\mathrm{S}$ alleles exhibited a certain degree of polymorphism, and the fragments of different lengths could generally be obtained using degenerate primers. To obtain more feasible genotypes, we also designed degenerate primers for the relatively conserved regions of the two sequences using DNA as a template to test the materials listed in Table 2 again. The degenerate primer was designed against the third exon of the $S$-RNase, and the product of $S_{b}$-RNase was 9 bp larger than that of $S_{a}$-RNase. Green Taq ${ }^{\mathrm{TM}}$ Mix (Vazyme, Nanjing, China) was used in combination with degenerate primers $\mathrm{FS}_{\mathrm{a}} \mathrm{S}_{\mathrm{b}}$ and $\mathrm{RS}_{\mathrm{a}} \mathrm{S}_{\mathrm{b}}$ in the PCR. The amplification procedure was as follows: $95^{\circ} \mathrm{C}$ for 5 min and 36 cycles $\left(95^{\circ} \mathrm{C}\right.$ for $15 \mathrm{~s}, 60^{\circ} \mathrm{C}$ for $15 \mathrm{~s}$, and $72^{\circ} \mathrm{C}$ for $40 \mathrm{~s}$ ), a final extension step at $72{ }^{\circ} \mathrm{C}$ for $5 \mathrm{~min}$, and storage at $10^{\circ} \mathrm{C}$. The PCR product was diluted 10 times, and $2 \mu \mathrm{L}$ was examined after staining with bromophenol blue in a $10 \%$ polyacrylamide gel for electrophoresis. The running procedure was as follows: $90 \mathrm{v}$ pre-electrophoresis for $15 \mathrm{~min}$, $160 \mathrm{v}$ electrophoresis for $1.5 \mathrm{~h}$, pause for $30 \mathrm{~min}$, and 160 $\mathrm{v}$ electrophoresis again for $1.5 \mathrm{~h}$. The polyacrylamide gel was soaked in silver staining solution $\left(1 \mathrm{~g} \mathrm{AgNO}_{3}+\right.$ $\left.33 \mathrm{~mL} \quad \mathrm{C}_{2} \mathrm{H}_{5} \mathrm{OH}+3 \mathrm{~mL} \quad \mathrm{CH}_{3} \mathrm{COOH}+750 \mathrm{~mL} \quad \mathrm{ddH}_{2} \mathrm{O}\right)$ for $15 \mathrm{~min}$ and then soaked in a developer solution $(10 \mathrm{~g}$ $\mathrm{NaOH}+4.5 \mathrm{~mL} \mathrm{HCHO}+750 \mathrm{~mL} \mathrm{H}_{2} \mathrm{O}$ ) for more than 10 min after twice cleaning with $\mathrm{ddH}_{2} \mathrm{O}$ and subsequently photographed under white light. In addition to the selfing lines in Table 2, degenerate primers were also used to test the selfing progenies' $S$ genotypes, including S1-02-S2-30S3-09, S1-02-S2-76-S3-09, and S1-02-S2-76-S3-11, and the $S$ genotypes of the hybrid progenies between S1-02S2-30-S3-09 and S1-02-S2-76-S3-11.

\section{S-RNase expression analysis}

Using $F$. viridis 42 as the control, $S$-RNase expression in compatible strawberry species, such as $F$. vesca 41, $F$. nilgerrensis $45, F$. mandshurica 43 , and $F . \times$ ananassa "Benihoppe", was tested. Total RNA was extracted from the flower balls, and cDNA was synthesized for S-RNase expression detection. The detection primers were specific primers used to clone the full CDS of $S_{a}$ and $S_{b}$-RNase and their degenerate primers. At the same time, the styles, ovaries, and receptacles of the flower balls, as well as the petals, calyxes, pedicels, leaves, and anthers, were collected for tissue-specific expression analysis of $S_{a^{-}}$and $S_{b}$-RNase. Taking the unpollinated flower balls as the control, spatiotemporal expression analysis of $S$-RNase after pollination was performed at $6 \mathrm{~h}, 12 \mathrm{~h}, 18 \mathrm{~h}$, and $24 \mathrm{~h}$. The expression analysis of $S$-RNase in a different germplasm and different tissues was performed using RT-PCR, while spatiotemporal expression analysis was performed using qRT-PCR. The RT-PCR is detailed in the section "Cloning and sequence analysis of $S_{a}$-RNase and $S_{b}$-RNase", and the qRT-PCR method is detailed here. First, the synthesized cDNA was diluted to $100 \mathrm{ng} / \mu \mathrm{L}$ using SYBR Premix Ex TaqTM (TaKaRa), and then an $\mathrm{ABi} 7500$ fluorescent quantitative PCR system (Applied Biosystems, Bedford, MA, USA) was used to perform qRT-PCR. The internal reference primer was the elongation factor- $\alpha$ gene EF1- $\alpha$. Each reaction consisted of a $20 \mu \mathrm{L}$ volume containing $10.0 \mu \mathrm{L}$ SYBR Premix Ex TaqTM (TaKaRa), $1 \mu \mathrm{L}$ cDNA, $0.5 \mu \mathrm{L}$ of each primer $(10 \mu \mathrm{M})$, and $8 \mu \mathrm{L} \mathrm{ddH}_{2} \mathrm{O}$. The reaction conditions were as follows: $95^{\circ} \mathrm{C}$ for $4 \mathrm{~min}$ and 40 cycles $\left(95^{\circ} \mathrm{C}\right.$ for $20 \mathrm{~s}$, $60^{\circ} \mathrm{C}$ for $20 \mathrm{~s}$, and $72^{\circ} \mathrm{C}$ for $40 \mathrm{~s}$ ). The upstream and downstream primers were designed on the basis of the second and third exons of $S$-RNase, respectively. The primer 
pairs used to detect $S_{a}$-RNase were $\mathrm{FS}_{\mathrm{a}} \mathrm{k}$ and $\mathrm{RS} \mathrm{a}_{\mathrm{a}} \mathrm{k}$, and $\mathrm{RS}_{\mathrm{b}} \mathrm{k}$ and $\mathrm{FS}_{\mathrm{b}} \mathrm{k}$ were used to detect $S_{b}$-RNase (Supplementary Table S3). Each reaction had three technical replicates, and the $2^{-\Delta \Delta \mathrm{Ct}}$ method was used to evaluate the relative expression of genes.

\section{Pollination test and compatibility analysis of different hybridizations within and between species}

The anthers were removed from the large flower buds collected from 7 to 9 o'clock in the morning and were wrapped in sulfuric acid paper and placed in silica gel bottles. Afterward, they were stored in a $4^{\circ} \mathrm{C}$ refrigerator. The fully dried pollen was used in the pollination experiments or stored in a $-70^{\circ} \mathrm{C}$ refrigerator. Generally, on successive sunny days at warmer temperatures, strawberry plant buds (at stages C3-C5) were selected for artificial emasculation and isolated using pollination bags in the afternoon. On the following morning, starting at $9 \mathrm{am}$, artificial pollination was performed, and the flowers were isolated again. Generally, pollen with good germination capability, as determined using pollen activity tests, is used in pollination experiments. The pollen germination medium was slightly modified based on a previous method $^{45}$, and $0.01 \% \mathrm{GA}_{3}$ was added. Eleven flowers were selected for each set of pollination experiments, and one of the eleven flowers was collected $48 \mathrm{~h}$ after pollination to observe the growth status of pollen tubes in the style. The remaining flowers were generally removed from the pollination bags after pollination for $\sim 10 \mathrm{~d}$, and the pollen tube growth status in the style was observed with a previously described dyeing method ${ }^{45}$.

Receptacle development was observed, and the fruit-set and seed-set rates of single fruits were determined after $25 \mathrm{~d}$ via intraspecific pollination experiments. In addition, the (in)compatibility of different pollination hybridizations was analyzed by examining the overall growth status of the pollen tubes in the different styles. Three $F$. viridis lines were selected for use in the intraspecific pollination experiments for each $\mathrm{S}$ genotype, and one line from the different $\mathrm{S}$ genotypes was selected for use in the interspecific pollination experiments. In the interspecific hybridizations, all germplasms were self-compatible, excluding that of $F$. viridis. The flowering times of the different germplasms were slightly different, and the rate of development of the receptacle also varied across the germplasms. Typically, photographs of the fruit were taken $15-25 \mathrm{~d}$ after pollination, and the fruit-set rate following the interspecific hybridizations was determined; however, the seed-set rate of single fruits was determined after fruit maturity. In addition, for the intraspecific hybridization of $F$. viridis, mutual pollination was performed with blooming flowers of different S-genotype lines using the smearing method without the influence of external pollinators in the greenhouse, and the development and color changes of the receptacles and achenes were observed within $10 \mathrm{~d}$ of pollination.

\section{Collinearity analysis and $S$ locus location in the genomes of the genera Fragaria, Rosa, and Prunus}

Genome-wide collinearity analysis was performed between the $F$. vesca and rose and almond genomes as well as between the two rose genomes using BLAST, while MCScanX ${ }^{83}$ and Ciros (http://circos.ca/) were used to illustrate collinearity. The genomes and the associated versions used were as follows: $F$. vesca genome v4.0.a $1^{84}$, Rosa chinensis genome v1.0 (Rosa_v1) ${ }^{49}$, Rosa chinensis "Old Blush" homozygous genome v2.0 (Rosa_v2) ${ }^{85}$, and P. dulcis "Texas" genome v2.0. The chromosomes harboring the $S$ locus in almond and rose were used to further analyze collinearity with the whole genome of F. vesca to obtain the collinearity blocks distributed in F. vesca. Furthermore, based on whether the collinearity blocks in $F$. vesca exhibited collinearity with the $S$ locus of almond and rose, the numbers and positions of the $S$ loci in the genome of Fragaria were further determined. Based on the location of S-RNase in the reported almond genome, Rosa_v1 rose genome ${ }^{49}$, and the evaluated Rosa_v2 rose genome (Supplementary Fig. S13), the $S$ loci areas were obtained by extending the $3^{\prime}$ and 5 ' termini of $S$-RNase by $\sim 2.5 \mathrm{Mbp}$. The $F$. vesca genome regions that exhibited collinearity with the $S$ locus of the almond genome, Rosa_v1 rose genome, and Rosa_v2 rose genome were selected to perform a more accurate location analysis. The reason for compatibility in strawberry was further determined through a functional analysis of RNase T2 family members and the distribution of F-box family members at this locus.

\section{Acknowledgements}

Funding: This work was supported by the National Natural Science Foundation of China (Grant no. 32072540, 31872056), the Fundamental Research Funds for the Central Universities (Grant no. KYZZ2021002), and a project funded by the Priority Academic Program Development of Jiangsu Higher Education Institutions (PAPD).

\section{Author details}

${ }^{1}$ Laboratory of Fruit Crop Biotechnology, College of Horticulture, Nanjing Agricultural University, Nanjing 210095 Jiangsu, China. ${ }^{2}$ Laboratory of Genetics and Plant Breeding, Graduate School of Horticulture, Chiba University, Matsudo 271-8510 Chiba, Japan. ${ }^{3}$ Present address: Institute of Botany, Jiangsu Province and Chinese Academy of Sciences, Nanjing 210014 Jiangsu, China

\section{Author contributions}

Y.Q. and J.D. conceived the research and designed the experiments. C.G. and J.D. developed the plant materials, established the selfing lines of $F$. viridis, and performed basic data collection. J.D. performed most of the experiment, analyzed the data, and wrote the manuscript with the help of T.L., S.W., and Z.G. H.S. guided the completion of part of the experiment and provided technical support. Y.Q. and H.S. reviewed the manuscript. All authors reviewed and approved this submission.

Conflict of interest

The authors declare no competing interests. 
Supplementary information The online version contains supplementary material available at https://doi.org/10.1038/s41438-021-00623-x.

Received: 12 January 2021 Revised: 29 May 2021 Accepted: 1 June 2021 Published online: 01 August 2021

\section{References}

1. Evans, W. \& Jones, J. Incompatibility of Fragaria. Can. J. Genet. Cytol. 9, 831-836 (1967).

2. Evans, W. Evidence of a crossability barrier in diploid $x$ hexaploid and diploid $x$ octoploid crosses in the genus Fragaria. Euphytica 23, 95-100 (1974).

3. Ahmadi, H. \& Bringhurst, R. Breeding strawberries at the decaploid level. Amer. Soc. Horti. Sci. 117, 856-862 (1992).

4. Marta, A., Camadro, E., Díaz-Ricci, J. \& Castagnaro, A. Breeding barriers between the cultivated strawberry, Fragaria Xananassa, and related wild germplasm. Euphytica 136, 139 (2004).

5. Bors, R. \& Sullivan, J. Interspecific hybridization of $F$. vesca subspecies with $F$. nilgerrensis, F. nubicola, F. pentaphylla, and F. viridis. J. Am. Soc. Horticultural Sci. 130, 418-423 (2005)

6. Bosković, R., Sargent, D. \& Tobutt, R. Genetic evidence that two independent S-loci control RNase-based self-incompatibility in diploid strawberry. J. Exp. Bot. 61, 755-763 (2010).

7. Bedinger, P., Broz, A., Tovar-Mendez, A. \& McClure, B. Pollen-pistil interactions and their role in mate selection. Plant Physiol. 173, 79-90 (2017).

8. Qin, X. et al. A farnesyl pyrophosphate synthase gene expressed in pollen functions in S-RNase-independent unilateral incompatibility. Plant J. 93 417-430 (2018).

9. Fujii, S., Kubo, K. \& Takayama, S. Non-self-and self-recognition models in plant self-incompatibility. Nat. Plants 2, 16130 (2016).

10. Franklin-Tong, N. \& Franklin, F. Gametophytic self-incompatibility inhibits pollen tube growth using different mechanisms. Trends Plant Sci. 8, 598-605 (2003).

11. Kubo, K. et al. Collaborative non-self recognition system in S-RNase-based selfincompatibility. Science 330, 796-799 (2010).

12. Potter, D. et al. Phylogeny and classification of Rosaceae. Plant Syst. Evol. 266, 5-43 (2007)

13. Zhang, S. et al. Diversification of Rosaceae since the Late Cretaceous based on plastid phylogenomics. N. Phytologist. 214, 1355-1367 (2017).

14. Chen, X. et al. Molecular systematics of Rosoideae (Rosaceae). Plant Syst. Evol. 306, 9 (2020).

15. Claessen, H. et al. Finding a compatible partner: Self-incompatibility in European pear (Pyrus communis); molecular control, genetic determination, and impact on fertilization and fruit set. Front. Plant Sci. 10, 407 (2019).

16. Tao, R. \& lezzoni, A. The S-RNase-based gametophytic self-incompatibility system in Prunus exhibits distinct genetic and molecular features. Sci. Horticulturae. 124, 423-433 (2010)

17. $\mathrm{Wu}, \mathrm{J}$. et al. Molecular determinants and mechanisms of gametophytic selfincompatibility in fruit trees of Rosaceae. Crit. Rev. Plant Sci. 32, 53-68 (2013).

18. Aguiar, B. et al. Convergent evolution at the gametophytic self-incompatibility system in Malus and Prunus. PLoS ONE 10, e0126138 (2015).

19. Sassa, H. Molecular mechanism of the S-RNase-based gametophytic selfincompatibility in fruit trees of Rosaceae. Breed. Sci. 66, 116-121 (2016).

20. Sassa, H., Hirano, H., Nishio, T. \& Koba, T. Style-specific self-compatible mutation caused by deletion of the S-RNase gene in Japanese pear (Pyrus serotina). Plant J. 12, 223-227 (1997).

21. Sonneveld, T., Tobutt, K., Vaughan, S. \& Robbins, T. Loss of pollen-S function in two self-compatible selections of Prunus avium is associated with deletion/ mutation of an S haplotype-specific F-box gene. Plant Cell. 17, 37-51 (2005).

22. Liang, M. et al. Evolution of self-compatibility by a mutant $S m$-RNase in citrus. Nat. Plants 6, 131-142 (2020)

23. Li, Y. et al. A mutation near the active site of S-RNase causes self-compatibility in S-RNase-based self-incompatible plants. Plant Mol. Biol. 103, 129-139 (2020).

24. Qin, X., Liu, B., Soulard, J. \& Morse, D. \& Cappadocia, M. Style-by-style analysis of two sporadic self-compatible Solanum chacoense lines supports a primary role for S-RNases in determining pollen rejection thresholds. J. Exp. Bot. 57, 2001-2013 (2006).

25. Shi, D. et al. Transcriptome and phytohormone analysis reveals a comprehensive phytohormone and pathogen defence response in pear self-/crosspollination. Plant Cell Rep. 36, 1785-1799 (2017).
26. Sanzol, J. Pistil-function breakdown in a new S-allele of European pear, $\mathrm{S}_{21}{ }^{\circ}$ confers self-compatibility. Plant Cell Rep. 28, 457-467 (2009).

27. Fernández i Marti, A., Gradziel, T. \& Socias i Company, R. Methylation of the $S_{f}$ locus in almond is associated with S-RNase loss of function. Plant Mol. Biol. 86, 681-689 (2014)

28. Lewis, D. \& Crowe, L. K. Unilateral interspecific incompatibility in flowering plants. Heredity 12, 233-256 (1958).

29. Liston, A., Cronn, R. \& Ashman, T. Fragaria: a genus with deep historical roots and ripe for evolutionary and ecological insights. Am. J. Bot. 101, 1686-1699 (2014).

30. Hancock, J.iln: Strawberries. 25-46 (CABI Publishing,1999).

31. Sargent, D. et al. Quantitative and qualitative differences in morphological traits revealed between diploid Fragaria species. Ann. Bot. 94, 787-796 (2004).

32. Ramanauskas, K. \& Igić, B. The evolutionary history of plant T2/S-type ribonucleases. Peer」 5, e3790 (2017).

33. Meng, D. et al. Apple MdABCF assists in the transportation of S-RNase into pollen tubes. Plant J. 78, 990-1002 (2014).

34. Meng, D. et al. The microtubule cytoskeleton and pollen tube Golgi vesicle system are required for in vitro S-RNase internalization and gametic selfincompatibility in apple. Plant Cell Physiol. 55, 977-989 (2014).

35. Sassa, $H$. et al. Self-incompatibility $(S)$ alleles of the Rosaceae encode members of a distinct class of the T 2/S ribonuclease superfamily. Mol. Gen. Genet. 250 547-557 (1996).

36. Ishimizu, $\mathrm{T}$. et al. Identification of regions in which positive selection may operate in S-RNase of Rosaceae: Implication for S-allele-specific recognition sites in S-RNase. FEBS Lett. 440, 337-342 (1998).

37. Ushijima, K. et al. Cloning and characterization of cDNAs encoding S-RNases from almond (Prunus dulcis): primary structural features and sequence diversity of the S-RNases in Rosaceae. Mol. Gen. Genet. 260, 261-268 (1998).

38. Yaegaki, H. et al. Molecular characterization of S-RNase genes and S-genotypes in the Japanese apricot (Prunus mume Sieb. et Zucc.). Sex. Plant Reprod. 13, 251-257 (2001).

39. Roalson, E. \& McCubbin, A. S-RNases and sexual incompatibility: structure, functions, and evolutionary perspectives. Mol. Phylogenet. Evol. 29, 490-506 (2003).

40. Banović, B., Šurbanovski, N., Konstantinović, M. \& Maksimović, V. Basic RNases of wild almond (Prunus webbii): cloning and characterization of six new SRNase and one "non-S RNase" genes. J. Plant Physiol. 166, 395-402 (2009).

41. Yamane, H., Ikeda, K., Ushijima, K., Sassa, H. \& Tao, R. A pollen-expressed gene for a novel protein with an F-box motif that is very tightly linked to a gene for S-RNase in two species of cherry, Prunus cerasus and P. avium. Plant Cell Physiol. 44, 764-769 (2003).

42. McCubbin, A. \& Kao, T. Molecular recognition and response in pollen and pisti interactions. Annu. Rev. Cell Developmental Biol. 16, 333-364 (2000).

43. Zhu, X. et al. Comprehensive genomic analysis of the RNase T2 gene family in Rosaceae and expression analysis in Pyrus bretschneideri. Plant Syst. Evolution. 306, 71 (2020).

44. Heng, W. et al. Identification of S-genotypes and novel S-RNase alleles in Prunus mume. J. Hortic. Sci. Biotechnol. 83, 689-694 (2008).

45. Du, J. et al. Identifying genome-wide sequence variations and candidate genes implicated in self-incompatibility by resequencing Fragaria viridis. Int. J. Mol. Sci. 20, 1039 (2019).

46. Chen, F., Dong, W., Zhang, J., Guo, X., Chen, J., Wang, Z., Lin, Z., Tang, H. \& Zhang, $L$. The sequenced angiosperm genomes and genome databases. Front. Plant Sci. 9, 418 (2018).

47. Chen, F., Song, Y., Li, X., Chen, J., Mo, L., Zhang, X., Lin, Z. \& Zhang, L. Genome sequences of horticultural plants: past, present, and future. Hortic. Res. 6, 112 (2019).

48. Gar, O. et al. An autotetraploid linkage map of rose (Rosa hybrida) validated using the strawberry (Fragaria vesca) genome sequence. PLOS ONE 6, e20463 (2011).

49. Hibrand Saint-Oyant, L. et al. A high-quality genome sequence of Rosa chinensis to elucidate ornamental traits. Nat. Plants 4, 473-484 (2018).

50. Staudt, G. Strawberry biogeography, genetics and systematics. Acta Hortic 842, 71-84 (2009)

51. Vieira, J., Pimenta, J., Gomes, A., Laia, J., Rocha, S., Heitzler, P. \& Vieira, C. P. The identification of the Rosa S-locus and implications on the evolution of the Rosaceae gametophytic self-incompatibility systems. Sci. Rep. 11, 3710 (2021).

52. Baek, Y. et al. Testing the SI $\times$ SC rule: pollen-pistil interactions in interspecific crosses between members of the tomato clade (Solanum section Lycopersicon, Solanaceae). Am. J. Bot. 102, 302-311 (2015). 
53. Zhang, X., Li, B., Zhao, Z., Guo, S. \& Qi, G. Relationship between compatibility of self-pollination and changes in protecting enzyme and hormone in different apple cultivars. Sci. Silvae Sin. 45, 20-25 (2009). (in Chinese).

54. Abdallah, D., Baraket, G., Perez, V., Hannachi, A. \& Hormaza, J. Self-compatibility in peach [Prunus persica (L.) Batsch]: patterns of diversity surrounding the Slocus and analysis of SFB alleles. Hortic. Res. 7, 170 (2020).

55. Ushijima, K. et al. The S haplotype-specific F-box protein gene, SFB, is defective in self-compatible haplotypes of Prunus avium and P. mume. Plant J. 39, 573-586 (2004).

56. Okada, K. et al. Deletion of a $236 \mathrm{~kb}$ region around $\mathrm{S}_{4}-\mathrm{RNase}$ in a stylarpart mutant $\mathrm{S}_{4}{ }^{\text {Sm }}$-haplotype of Japanese pear. Plant Mol. Biol. 66, 389-400 (2008).

57. Okada, K. et al. Related polymorphic F-box protein genes between haplotypes clustering in the BAC contig sequences around the S-RNase of Japanese pear. J. Exp. Bot. 62, 1887-1902 (2011).

58. Cachi, A. \& Wünsch, A. Characterization of self-compatibility in sweet cherry varieties by crossing experiments and molecular genetic analysis. Tree Genet. Genomes 10, 1205-1212 (2014).

59. Dissanayake, D. M. R. K. K., Norioka, S., Norioka, N., Takasaki, T. \& Nakanishi, T. Cisregulatory elements for pistil specific expression in $S$-RNase promoter region of Japanese pear (Pyrus pyrifolia Nakai). Acta Hortic. 587, 459-465 (2002).

60. De Franceschi, P., Bianco, L., Cestaro, A., Dondini, L. \& Velasco, R. Characterization of 25 full-length S-RNase alleles, including flanking regions, from a pool of resequenced apple cultivars. Plant Mol. Biol. 97, 279-296 (2018).

61. Wang, S., Kakui, H., Kikuchi, S., Koba, T. \& Sassa, H. Interhaplotypic heterogeneity and heterochromatic features may contribute to recombination suppression at the S locus in apple (Malus xdomestica). J. Exp. Bot. 63, 4983-4990 (2012).

62. Peng, J. \& Karpen, G. Epigenetic regulation of heterochromatic DNA stability. Curr. Opin. Genet. Dev. 18, 204-211 (2008).

63. Brink, M., Diewertje, P., Mateos-Langerak, J., Driel, R. \& Verschure, P. Chromosome organization and epigenetic gene regulation of the eukaryotic genome. 16th International Chromosome Conference (ICC 16) (2007).

64. Dickerson, D. et al. High resolution imaging reveals heterogeneity in chromatin states between cells that is not inherited through cell division. BMC Cell Biol. 17, 33 (2016)

65. Zuckerkandl, E. \& Junk, D. N. A. and sectorial gene repression. Gene 205 323-343 (1997).

66. Martianov, I., Ramadass, A., Barros, A., Chow, N. \& Akoulitchev, A. Repression of the human dihydrofolate reductase gene by a non-coding interfering transcript. Nature 445, 666-670 (2007).

67. Parenteau, J. \& Abou Elela, S. Introns: Good day junk is bad day treasure. Trends Genet. 35, 923-934 (2019).
68. Lewis, D. \& Crowe, L. Unilateral interspecifific incompatibility in flowering plants. Heredity 12, 232-256 (1958).

69. Liedl, B., McCormick, S. \& Mutschler, M. Unilateral incongruity in crosses involving Lycopersicon pennellii and $L$. esculentum is distinct from selfincompatibility in expression, timing and location. Sex. Plant Reprod. 9, 299-308 (1996).

70. Covey, P. et al. Multiple features that distinguish unilateral incongruity and selfincompatibility in the tomato clade. Plant J. 64, 367-378 (2010).

71. Chen, J. et al. Phosphatidic acid counteracts S-RNase signaling in pollen by stabilizing the actin cytoskeleton. Plant Cell. 30, 1023-1039 (2018).

72. De Nettancourt, D. Incompatibility and incongruity in wild and cultivated plants. Springer Sci. Bus. Media 3, 1-185 (2001).

73. Sun, J. et al. Complete chloroplast genome sequencing of ten wild Fragaria species in China provides evidence for phylogenetic evolution of Fragaria. Genomics 113, 1170-1179 (2021).

74. Morimoto, T., Akagi, T. \& Tao, R. Phylogenetic analysis of S-RNase-like genes from Fragaria provides evidence for ancestral S-RNase duplication in the Rosaceae lineage. Acta Hortic. 1235, 365-370 (2019).

75. Bourke, P. et al. Partial preferential chromosome pairing is genotype dependent in tetraploid rose. Plant J. 90, 330-343 (2017)

76. Finn, R., Clements, J. \& Eddy, S. HMMER web server: interactive sequence similarity searching. Nucleic Acids Res. 39, W29-W37 (2011).

77. Artimo, P. et al. ExPASy: SIB bioinformatics resource portal. Nucleic Acids Res. $\mathbf{4 0}$ W597-W603 (2012).

78. Armenteros, J. et al. SignalP 5.0 improves signal peptide predictions using deep neural networks. Nat. Biotechnol. 37, 420-423 (2019).

79. Stewart, $Z$. et al. Transcriptomic investigation of wound healing and regeneration in the cnidarian Calliactis polypus. Sci. Rep. 7, 41458 (2017).

80. Grabherr, M. et al. Trinity: reconstructing a full-length transcriptome without a genome from RNA-Seq data. Nat. Biotechnol. 29, 644 (2011).

81. Mello, B., Tao, Q., Tamura, K. \& Kumar, S. Fast and accurate estimates of divergence times from big data. Mol. Biol. Evol. 34, 45-50 (2017).

82. Luo, R. et al. SOAPdenovo2: an empirically improved memory-efficient shortread de novo assembler. Gigascience 1, 18 (2012).

83. Wang, Y. et al. MCScanX: a toolkit for detection and evolutionary analysis of gene synteny and collinearity. Nucleic Acids Res. 40, e49 (2012).

84. Edger, $P$. et al. Single-molecule sequencing and optical mapping yields an improved genome of woodland strawberry (Fragaria vesca) with chromosome-scale contiguity. GigaScience 7, gix124 (2018).

85. Raymond, O. et al. The Rosa genome provides new insights into the domestication of modern roses. Nat. Genet. 50, 772-777 (2018). 